\title{
A Snapshot of the Sun Near Solar Minimum: The Whole Heliosphere Interval
}

\author{
Barbara J. Thompson • Sarah E. Gibson · Peter C. Schroeder • David F. Webb • \\ Charles N. Arge · Mario M. Bisi - Giuliana de Toma • Barbara A. Emery • \\ Antoinette B. Galvin • Deborah A. Haber · Bernard V. Jackson • Elizabeth A. Jensen • \\ Robert J. Leamon • Jiuhou Lei • Periasamy K. Manoharan • M. Leila Mays • \\ Patrick S. McIntosh • Gordon J.D. Petrie • Simon P. Plunkett • Liying Qian • \\ Peter Riley • Steven T. Suess • Munetoshi Tokumaru • Brian T. Welsch • \\ Thomas N. Woods
}

Received: 13 September 2011 / Accepted: 3 November 2011 / Published online: 24 November 2011

(C) The Author(s) 2011. This article is published with open access at Springerlink.com

Abstract We present an overview of the data and models collected for the Whole Heliosphere Interval, an international campaign to study the three-dimensional solar-

Invited Review

The Sun-Earth Connection near Solar Minimum

Guest Editors: M.M. Bisi, B.A. Emery, and B.J. Thompson

B.J. Thompson

NASA Goddard Space Flight Center, Greenbelt, MD, USA

e-mail: Barbara.J.Thompson@nasa.gov

S.E. Gibson $(\varangle) \cdot$ G. de Toma $\cdot$ B.A. Emery $\cdot$ L. Qian

High Altitude Observatory, National Center for Atmospheric Research, Boulder, CO, USA

e-mail: sgibson@ucar.edu

P.C. Schroeder · B.T. Welsch

Space Sciences Laboratory, University of California, Berkeley, CA, USA

D.F. Webb

ISR, Boston College, Newton, MA, USA

C.N. Arge

Hanscom AFB Research Laboratory, Hanscom, MA, USA

M.M. Bisi · B.V. Jackson · E.A. Jensen

Center for Astrophysics and Space Sciences, University of California San Diego, La Jolla, CA, USA

M.M. Bisi

Institute of Mathematics and Physics, Aberystwyth University, Aberystwyth, Wales, UK

A.B. Galvin

EOS, University of New Hampshire, Durham, NH, USA

D.A. Haber

JILA, University of Colorado, Boulder, CO, USA 
heliospheric-planetary connected system near solar minimum. The data and models correspond to solar Carrington Rotation 2068 (20 March - 16 April 2008) extending from below the solar photosphere, through interplanetary space, and down to Earth's mesosphere. Nearly 200 people participated in aspects of WHI studies, analyzing and interpreting data from nearly 100 instruments and models in order to elucidate the physics of fundamental heliophysical processes. The solar and inner heliospheric data showed structure consistent with the declining phase of the solar cycle. A closely spaced cluster of low-latitude active regions was responsible for an increased level of magnetic activity, while a highly warped current sheet dominated heliospheric structure. The geospace data revealed an unusually high level of activity, driven primarily by the periodic impingement of high-speed streams. The WHI studies traced the solar activity and structure into the heliosphere and geospace, and provided new insight into the nature of the interconnected heliophysical system near solar minimum.

\section{Introduction}

The Whole Heliosphere Interval (WHI) was an internationally coordinated observation and modeling effort to characterize the three-dimensional (3D) interconnected solar-

Present address:

E.A. Jensen

Planetary Science Institute, Tucson, AZ, USA

R.J. Leamon

Montana State University, Bozeman, MT, USA

J. Lei

School of Earth and Space Sciences, University of Science and Technology of China, Hefei, China

P.K. Manoharan

Radio Astronomy Centre, Tata Institute of Fundamental Research, Ooty, Tamil Nadu, India

M.L. Mays

ORAU at NASA Goddard Space Flight Center, Greenbelt, MD, USA

P.S. McIntosh

Heliosynoptics, Inc., Boulder, CO, USA

G.J.D. Petrie

National Solar Observatory, Tucson, AZ, USA

S.P. Plunkett

Naval Research Laboratory, Washington, DC, USA

P. Riley

Predictive Science, San Diego, CA, USA

S.T. Suess

National Space Science and Technology Center, 320 Sparkman Drive, Huntsville, AL, USA

M. Tokumaru

STELab, Nagoya University, Nagoya, Japan

T.N. Woods

LASP, University of Colorado, Boulder, CO, USA 
heliospheric-planetary system, known as the "heliophysical" system. WHI focused on a single solar rotation: Carrington Rotation 2068 (CR 2068), which ran from 20 March16 April 2008. This solar rotation was the "origin" of the 3D structures and interactions studied by WHI. The data collected to study CR 2068 spanned a greater temporal range, in order to trace the solar wind's flow from the Sun through the heliosphere.

WHI was the largest Coordinated Investigation Program (CIP: Thompson et al., 2009) of the International Heliophysical Year (IHY) (2007-2009). IHY was inspired by the 50th Anniversary of the International Geophysical Year (1957-1958) and the subsequent 50 years of space exploration. IGY included the launch of Sputnik (the first spacecraft) and Explorer I, which brought the first scientific observations of space. Since then, there have been striking improvements in observations, including new 3D measurements, higher temporal/spatial/spectral resolutions, and expanded synoptic observations ranging from deep in the solar interior into the heliosphere and geospace. WHI was able to take full advantage of the 50 years of scientific progress since IGY by coordinating state-of-the-art models and observations to address the entire interconnected heliophysical system.

WHI consisted of several phases: After the preliminary planning and observation phases, several months of coordinated effort led to the "WHI Data and Modeling Assessment Workshop" held 26-29 August 2008 in Boulder, Colorado, USA. The two purposes of the workshop were to serve as a catalyst for the assembly of the data and analysis tools, and initiate the coordinated analysis efforts between the WHI community of modelers and data experts. The second WHI workshop, held 10 - 13 November 2009 in Boulder, focused on the generation of scientific results.

One of the earliest findings of WHI was that, although initial forecasts predicted the solar minimum to occur in late 2006/early 2007, the Sun during WHI in Spring 2008 was still exhibiting structure consistent with the declining phase of Solar Cycle 23. This spurred the formation of the International Astronomical Union Division II Working Group on "Comparative Solar Minima" (http://ihy2007.org/IAUWG/WEBPAGES/IAUWG.shtml), and prompted the selection of two additional solar rotations to be added to certain elements of WHI studies: WHI 2 (corresponding to CR 2078, which ran from 17 December 2008 to 12 January 2009), and WHI 3 (corresponding to CR 2085, which ran from 26 June 2009 to 22 July 2009). For a more detailed description of the WHI 2 and WHI 3 campaign results, please see Gibson et al. (2011).

WHI studies included the origins and impact of both static and evolving structures extending from the solar interior, through the heliosphere and geospace, and into the Earth's atmosphere. In this article, we present the "WHI Mosaic", which is an assembly of maps and plots from dozens of observations and modeling efforts that represent the 3D structure of the Sun during the primary observation interval, CR 2068. This "snapshot" of the interconnected Sun-Earth system provides a backdrop for the research efforts described in this Topical Issue of Solar Physics: "The Sun-Earth Connection near Solar Minimum: Placing it into Context." The articles in this Topical Issue represent results from all of WHI's observational regimes, as well as research on the nature of the solar minimum in general.

\section{Scientific Motivation}

\subsection{The "New" Science of Heliophysics}

The term "heliophysical" came from a broadening of the concept "geophysical," extending the connections from the Earth to the Sun and into interplanetary space. As the International 
Heliophysical Year's largest research program, WHI incorporated the drivers of geophysical change into the extended solar-heliospheric domain. The primary science goals of WHI were to:

- Characterize the 3D solar minimum heliosphere

- Trace the effects of solar structure and activity through the solar wind to the Earth and other planetary systems.

To accomplish the first goal, synoptic observing programs were run throughout WHI, along with several "targeted observing campaigns" (e.g. Chamberlin et al., 2009; McIntosh and De Pontieu, 2009; Woods et al., 2009). These observations were interpreted and connected with the aid of models, providing a phenomenological framework. With the establishment of the framework, a variety of cross-disciplinary science questions could be addressed. The following science questions were identified for WHI. We take this opportunity to provide the relevant references to articles in this Topical Issue or other publications that specifically involved WHI:

i) What is the impact of a solar minimum "ground state", in particular a 3D solar magnetic field with predominantly open flux and a lack of solar activity, on

- Solar-driven Ionosphere-Thermosphere-Mesosphere coupling (including irradiancedriven processes)? (Chamberlin et al., 2009; Woods et al., 2009; Solomon et al., 2010, 2011; Araujo-Pradere et al., 2011; Emery et al., 2011; Gibson et al., 2011; Haberreiter, 2011; Lei et al., 2011; Wang et al., 2011; White et al., 2011)

- Cosmic-ray modulation? (Gibson et al., 2011; Jian, Russell, and Luhmann, 2011)

- Morphology of heliosphere? (Bisi et al., 2009a; Cliver and Ling, 2011; Echer, Tsurutani, and Gonzalez, 2011; Riley et al., 2011; Zhao and Fisk, 2011)

- Solar and magnetospheric magnetic energy storage/release? (Emery et al., 2011; Jian, Russell, and Luhmann, 2011; Lepping et al., 2010)

- And once we have answered these questions, does it help us interpret the historical record? (Cliver and Ling, 2011; Echer, Tsurutani, and Gonzalez, 2011; Emery et al., 2011; Gibson et al., 2009; Jian, Russell, and Luhmann, 2011)

ii) What processes determine the physics at heliophysical boundaries?

- What can we learn from composition boundaries (at Sun and in solar wind)? (Leamon and McIntosh, 2009; Gibson et al., 2011; Zhao and Fisk, 2011)

- What can we learn about plasma/neutral and density boundaries? (Echer, Tsurutani, and Gonzalez, 2011; Emmert, Lean, and Picone, 2010; Gibson et al., 2009, 2011; McIntosh and De Pontieu, 2009; de Toma, 2010; Lei et al., 2011; Wang et al., 2011; Vásquez et al., 2011)

- How about magnetic boundaries (current sheets/sector boundaries, magnetopauses)? (Petrie, Canou, and Amari, 2011; Riley et al., 2011; Zhao and Fisk, 2011)

- Is there evidence for interchange reconnections at coronal-hole boundaries? (Zhao and Fisk, 2011)

iii) How is magnetic energy transported through the system?

- How do solar subsurface structures/flow relate to magnetic flux emergence? (Kosovichev and Duvall, 2011; Altrock, 2011; Muller, Utz, and Hanslmeier, 2011; Webb et al., 2011) 
Figure 1 WHI's heliospheric observatories. In March 2008, Ulysses was completing its final polar pass as the STEREO mission was approaching $50^{\circ}$ separation. ACE, SOHO, and Wind were located near the Sun-Earth $\mathrm{L}_{1}$ point. (Graphic from Suess (2007) modified to indicate STEREO and Earth locations.)

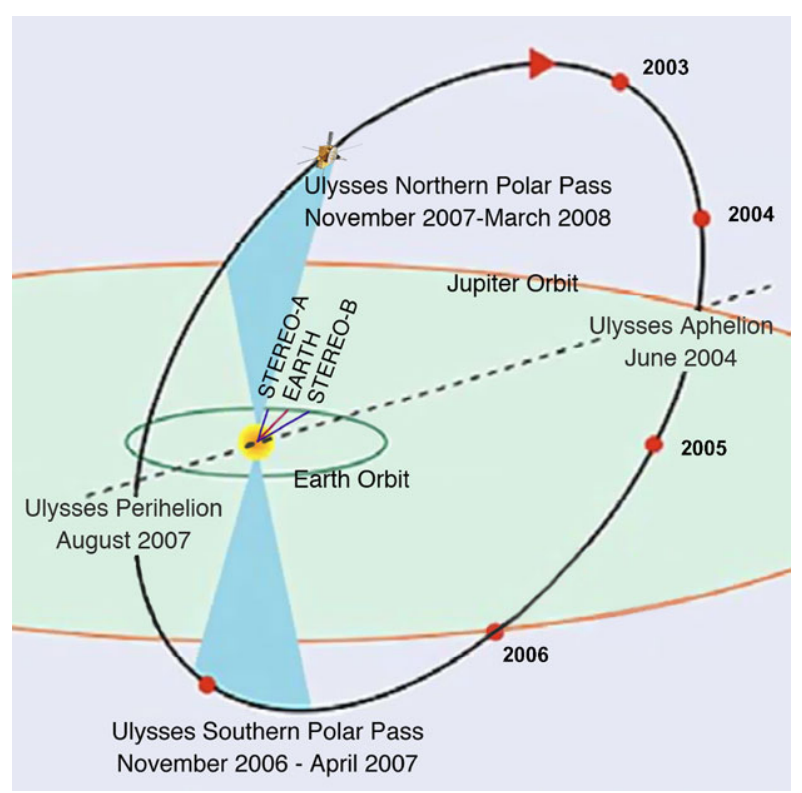

- How does flux emergence/reconnections/MHD waves affect evolution of solar magnetic structures? (Aschwanden 2011a, 2011b; Nitta, 2011; Petrie, Canou, and Amari, 2011; Vásquez et al., 2011; Welsch, Christe, and McTiernan, 2011)

- How do structures (both eruptive and non-eruptive) evolve in solar wind from Sun to heliopause? (Cremades, Mandrini, and Dasso, 2011; Lepping et al., 2010; Maris and Maris, 2010; Riley et al., 2011; Webb et al., 2011)

- How do they interact with planetary magnetospheres and geospace? (Araujo-Pradere et al., 2011; Echer, Tsurutani, and Gonzalez, 2011; Emery et al., 2011; Jackman and Arridge, 2011; Lei et al., 2011; Wang et al., 2011)

To properly address these questions, the dates of WHI needed to occur near solar minimum, and multi-point heliospheric measurements would have to include out-of-the-Ecliptic observations provided by the Ulysses mission. Fortunately, Ulysses was in an ideal location, and the STEREO mission provided two additional measurements points near the solar Ecliptic plane.

\subsection{Observational Vantage Points}

By Spring 2008, the field of heliophysics was entering a new era of 3D observing capabilities. Two recently launched multi-spacecraft missions (STEREO and THEMIS) were expanding our capacity to study variations in the global structure of the heliosphere and magnetosphere, respectively, while the extremely successful Ulysses mission was approaching the end of nearly two decades of out-of-the-Ecliptic observations. The Cassini and Voyager missions continued their measurements of the outer heliosphere, and a new mission to be launched in October 2008, the Interstellar Boundary Explorer (IBEX), would soon be mapping the boundary of the heliosphere itself. These factors, along with the continued operation of dozens of additional missions and observatories (both ground- and space-based), made Spring 2008 an excellent time for 3D heliospheric studies, and therefore an ideal occasion for the Whole Heliosphere Interval campaign. 


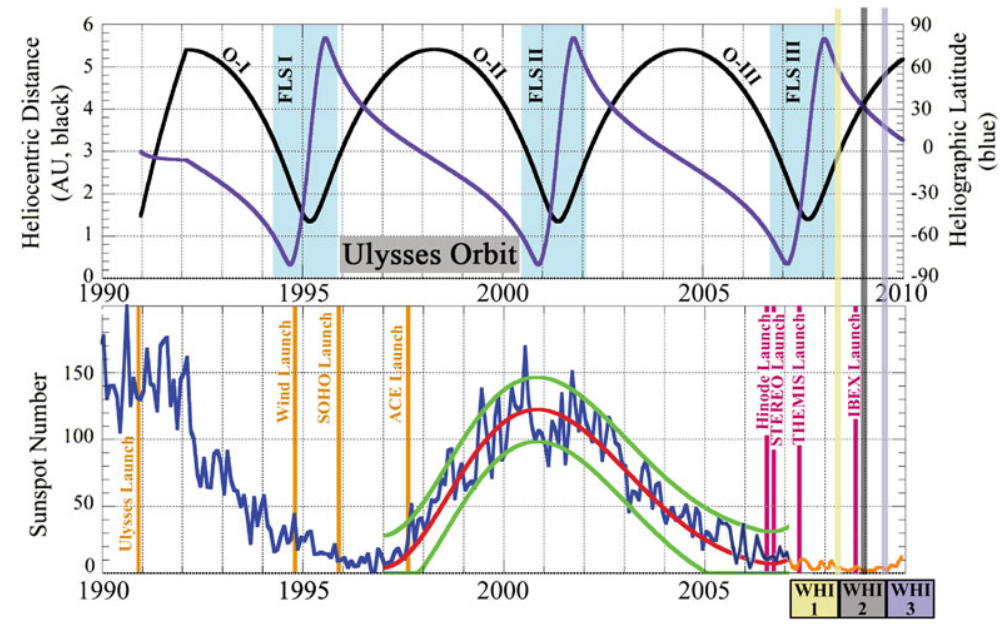

Figure 2 The date of the forecasted solar minimum (lower plot, red line $=$ mean forecast, green lines $=$ forecast envelope) coincided with the Ulysses mission's final high-latitude transit of the heliosphere (upper plot, blue line), as well as the launch of several new heliophysics missions and the preparation for the upcoming IBEX mission. Solar minimum occurred at the end of 2008, and the slow rise of Cycle 24 fell far outside the forecast's margin of error (green lines). Two supplementary intervals, WHI 2 and WHI 3, were later added to provide a more complete view of the current solar minimum. (Figure adapted from Suess (2007). Forecast data obtained in 2007 from D.H. Hathaway, private communication.)

In early 2008 the Ulysses mission was completing its final transit over the northern pole of the Sun. Throughout 2007, Ulysses was in the "Fast Latitude Scan" (FLS) phase of its orbit, where it is near the 1.4 AU perihelion of its highly elliptical orbit (see Figure 1 and top of Figure 2). During each FLS, Ulysses traveled from $80^{\circ}$ below the Ecliptic to $80^{\circ}$ above in less than one-fifth of the time it took to complete the rest of an orbit.

The STEREO spacecraft, launched in October 2006, were approaching $50^{\circ}$ separation. The Sun-Earth $\mathrm{L}_{1}$ Lagrange point, with $\mathrm{SOHO}$, ACE, and Wind, was roughly halfway between the two. The three measurement points near the solar Ecliptic plane, plus the Ulysses measurement out of the Ecliptic, made it possible to study the evolution of the 3D heliosphere in greater detail than ever before. The angular separation between the STEREO spacecraft was still small enough to perform stereoscopic imaging, and the distance between the two spacecraft was still within the size of an average coronal mass ejection (CME) or a large corotating interaction region/stream interaction region (CIR/SIR), thus making it possible to study heliospheric structures with multiple sampling points.

Another key WHI observational asset, launched 14 April 2008, was a sounding rocket experiment that enabled the creation of the first complete high-resolution irradiance spectrum near solar minimum conditions (Chamberlin et al., 2009). NASA sounding rocket 36.240 (the Woods/LASP rocket) obtained EUV measurements that, when combined with data from TIMED/SEE and SORCE, covered the full spectral range from the EUV to infrared $(0.1 \mathrm{~nm}$ to $2400 \mathrm{~nm}$ ). The production of the new Solar Irradiance Reference Spectra (SIRS: Woods et al., 2009), was a major effort by many members of the WHI team, and constitutes one of WHI's most significant scientific accomplishments.

\subsection{Heliophysics at (and near) Solar Minimum}

The Whole Heliosphere Interval campaign occurred near solar minimum, in order to optimize our ability to characterize the $3 \mathrm{D}$ heliosphere and trace the connections to geospace 

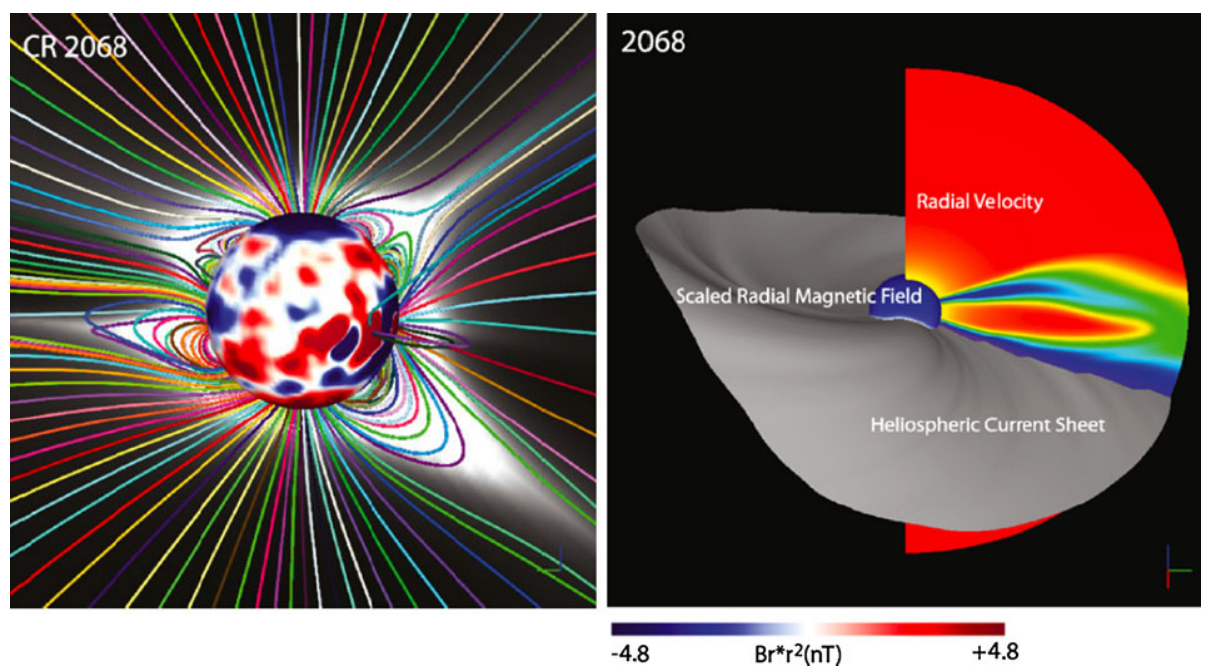

Figure 3 WHI's "atypical" solar minimum structure. Left: Composite image of a modeled photospheric magnetic field at the solar surface (saturated at $\pm 1 \mathrm{G}$ ), with a selection of magnetic-field lines originating in the plane of the figure, and a color contour of the coronal density (scaled by $R^{2}$ ). Right: Illustration of the smoothed large-scale properties of the inner heliosphere out to $1 \mathrm{AU}$. The isosurface marks the location of $B_{r}=0$ and is the location of the heliospheric current sheet (HCS). The meridional slice shows the modeled radial velocity, and the sphere at $30 R_{\odot}$ shows the radial magnetic-field strength. (Figures from Riley et al. (2011).)

and to the outer heliosphere. WHI was based on, and grew out of, the extremely successful "Whole Sun Month" (WSM) campaigns that took place during the previous solar minimum (e.g. Biesecker et al., 1999; Galvin and Kohl, 1999; Gibson et al., 1999; Linker et al., 1999). The comprehensive suite of observations and models allowed a thorough characterization of the solar-minimum heliosphere. WHI expanded on WSM by including geospace, and including a broader and more sophisticated range of models and observations, especially of the heliosphere. It was presumed that, for the second consecutive solar cycle, WHI would allow researchers a deeper look into the nature of a "typical" solar minimum. Giving consideration to the observatory orbit locations, observing schedules, and forecasts for the minimum of Solar Cycle 23 (see Figure 2), the WHI team chose Carrington Rotation 2068, running 20 March - 16 April 2008.

As it turned out, the minimum between Solar Cycles 23 and 24 was quite different from other recent minima (e.g. Gibson et al., 2009, 2011). In March 2007, a panel of forecasters on the NOAA/NASA/ISES Solar Cycle 24 Prediction Panel expressed "high" confidence that solar minimum would occur in early 2008. In actuality, solar minimum occurred in December 2008, and it was the longest and quietest minimum in a century. In 2008, no sunspots were observed on 266 of the year's 366 days (73\%). 2009 had a similar number of spotless days (71\%), placing both 2008 and 2009 in the top-five sunspotless years since 1849.

Due to the unanticipated delay in the arrival of Solar Cycle 24, WHI transpired more than six months before the cycle reached the actual minimum, and the solar structure during WHI was much more complex than during the Whole Sun Month (see Figure 3). Nonetheless, the data and models assembled as part of WHI allowed a great deal of insight into the nature of the heliophysical connected system near solar minimum, and the addition of the WHI 2 and WHI 3 intervals allowed a supplementation of data to provide a more complete picture. 


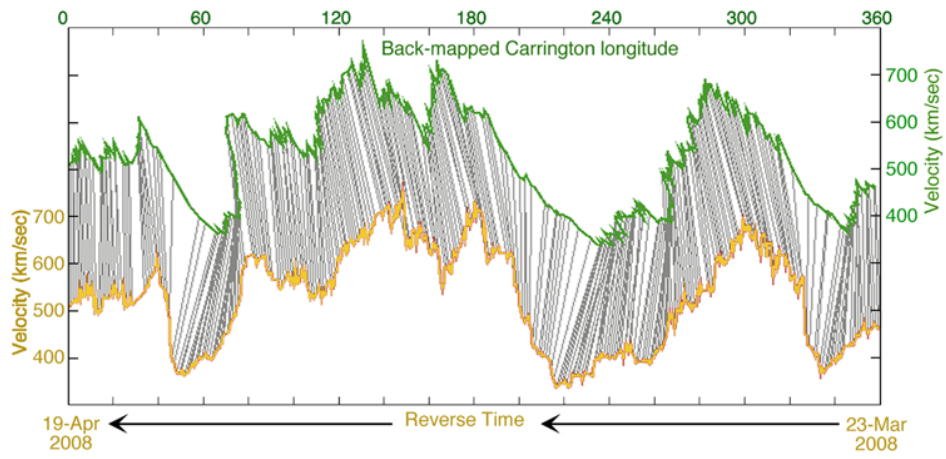

Figure 4 Projecting solar wind data back to the solar source longitude. In order to compare the solar-wind and geospace data with their associated solar structures, the in-situ data are mapped back to the estimated source surface $\left(R=2.5 R_{\odot}\right)$ longitude of the Sun based on the observed solar-wind speed. As with the Carrington maps, time goes "backward," and decreases along the $x$-axis. Shown is the Wind/SWE solar-wind speed with the temporal axis reversed (orange), lower and left axes) and back-projected as a function of Carrington longitude (green, right and upper axes).

\section{WHI Synoptic Data and Models}

Most of the observatories participating in WHI compiled datasets consisting of "synoptic" observations, which were designed to provide baseline measurements of the heliophysical system. The same measurements were performed over the course of the entire campaign, building up a dataset representing an entire solar rotation. The data and models presented in Figures 5-11 represent these synoptic studies.

The solar and inner-heliospheric data are presented in the form of Carrington maps. The $y$-axis of the Carrington maps indicates heliographic latitude, and the $x$-axis corresponds to Carrington longitude (CLON). CR 2068 began at the 01:18 UT on 20 March 2008, when $\mathrm{CLON}=360$ was at the solar meridian, as viewed from Earth. The longitudes count down in time until CLON $=0$ occurs at 08:09 UT on 16 April 2008. This also coincides with the beginning of the new rotation, $C R 2069, \mathrm{CLON}=360$. (Note: the corresponding dates are different for missions outside of Earth's orbit.)

The in-situ and geospace data are presented using a coordinate system that facilitates comparison to the solar and heliospheric Carrington maps. During WHI, the solar-wind speed measured by the spacecraft near the Ecliptic plane ranged from 350 to $750 \mathrm{~km} \mathrm{~s}^{-1}$. At these velocities, the transit from the Sun to $1 \mathrm{AU}$ takes between $\approx 2.5$ to 5 days, with a mean time of about 3.5 days (which corresponds to approximately $45^{\circ}$ in Carrington longitude). The "back-mapping" representation takes into account the several days of separation between the observation of a solar feature and in-situ detection of any associated solar-wind structures.

The back-mapping technique is illustrated in Figure 4. In this article, all of the geospace and in-situ measurements are mapped back to their estimated source longitude at the solar source surface $\left(R=2.5 R_{\odot}\right)$, based on the local measured in-situ solar-wind speed. In Figure 4, the lower and left axes (which are colored orange) show the solar-wind speed as measured by Wind/SWE, plotted as a function of time, only with the $x$-axis running backward in time (right to left) instead of forward. The green line (upper and right axes) show the same data as the orange line, but instead of plotting as a function of time, the data are plotted as a function of Carrington source longitude. The thin black lines between the orange 

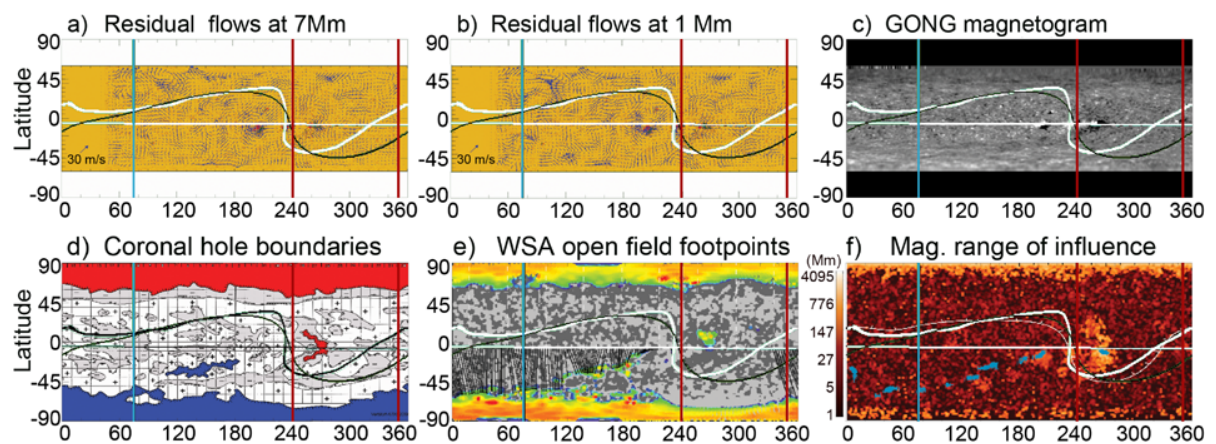

g) Observed coronal holes
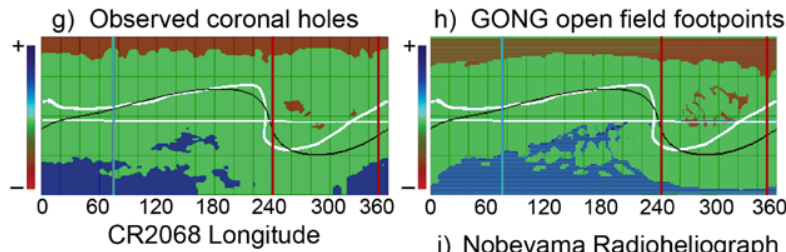

i) MAS open field footpoints
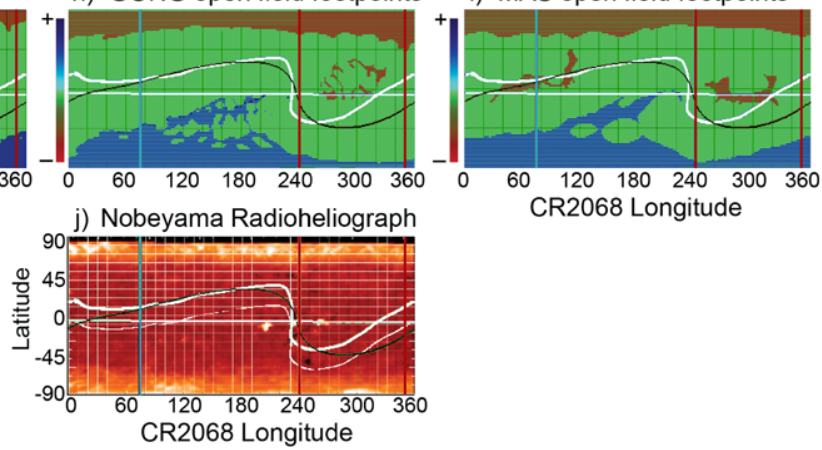

CR2068 Longitude

Figure 5 Solar Carrington maps for subsurface, magnetograph, radio, coronal hole, magnetic range of influence, and solar-wind footpoint data. The maps correspond to the following: (a) residual subsurface flow map from $7 \mathrm{Mm}$ below the photosphere; (b) residual subsurface flow map from $1 \mathrm{Mm}$ below the photosphere; (c) GONG longitudinal magnetogram; (d) coronal-hole boundaries and polarities; (e) WSA model magnetic-field and open-field footpoints; (f) magnetic range of influence (MRoI); (g) coronal holes identified in EUV data; (h) open-field footpoints from PFSS extrapolation of GONG data; (i) open-field footpoints from MAS model extrapolation; (j) Nobeyama 17-GHz radio emission map.

and green plots correspond connect the in-situ (orange) measurements to their back-mapped (green) locations.

Of course, the solar-wind speed is rarely constant, and this simple "ballistic" estimate is not physically realistic, but it is useful to facilitate comparison. Because slower-speed wind tends to speed up as it interacts with higher-speed solar wind, the ballistically mapped-back longitude tends to be greater than the actual source longitude (the opposite being true for faster wind). As a result, the speeds for a given longitude are not necessary single-valued (as is evident near $\mathrm{CLON}=75^{\circ}$ ), and these regions have an increased likelihood of developing into fast/slow stream-interaction regions (SIRs) or corotating interaction regions (CIRs).

\subsection{Solar Carrington Maps}

Figures 5 and 6 show the data from solar imagers and models. The solar-disk imager data are interpolated where there is adequate coverage, and the maps are left blank where the coverage is incomplete.

There are several pieces of supplementary information superposed on each of the maps. These include $i$ ) the location of the Earth relative to the solar Ecliptic plane (heliolatitude), ii) the times of two heliospheric current sheet (HCS) crossings, iii) a CIR with an ICME observed at $\mathrm{L}_{1}$, and $i v$ ) the heliospheric source-surface location according to two different models. 

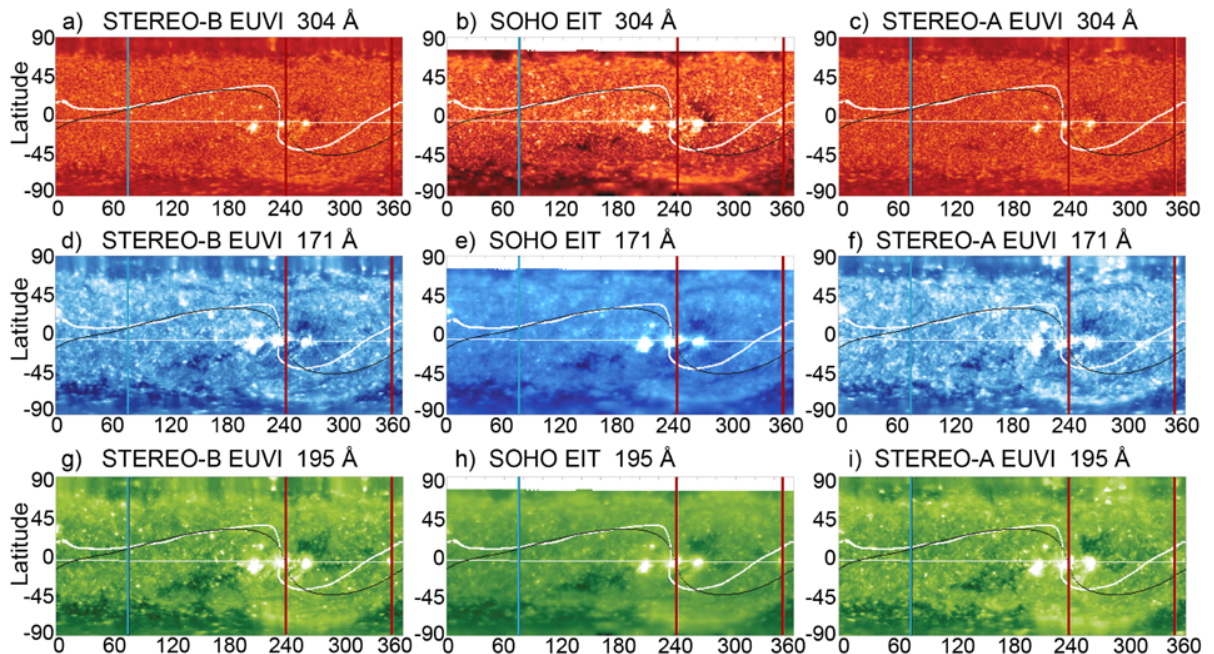

h) SOHO EIT $195 \AA$

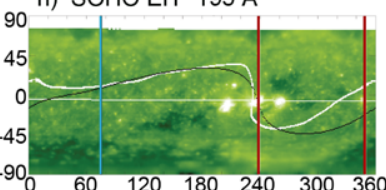

i) STEREO-A EUVI $195 \AA$
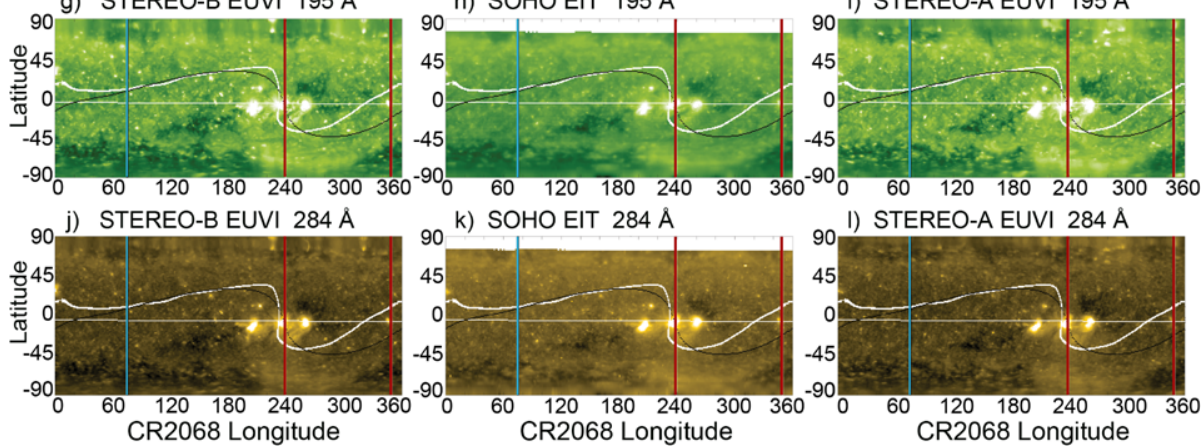

Figure 6 Solar Carrington maps of data from EUV imagers. (a) $304 \AA$ map constructed from STEREO-B/SECCHI/EUVI data; (b) $304 \AA$ map from SOHO/EIT; (c) $304 \AA$ map from STEREO-A/SECCHI/EUVI; (d) $171 \AA$ map from STEREO-B/SECCHI/EUVI; (e) $171 \AA$ map from SOHO/EIT; (f) $171 \AA$ map from STEREO-A/SECCHI/EUVI; (g) $195 \AA$ map from STEREO-B/ SECCHI/EUVI; (h) $195 \AA$ map from SOHO/EIT; (i) $195 \AA$ map from STEREO-A/SECCHI/EUVI; (j) $284 \AA$ map from STEREO-B/SECCHI/EUVI; (k) $284 \AA$ map from SOHO/EIT; (1) $284 \AA$ map from STEREO-A/SECCHI/EUVI.

i) Earth's heliolatitude/ $B$-angle $\left(B_{0}\right)$ : Throughout WHI, the Earth was located South of the solar equatorial plane, ranging from $B_{0}=-7.03^{\circ}$ on 20 March 2008 to $B_{0}=-5.48^{\circ}$ on 16 April 2008. The solar and heliospheric Carrington maps in Figures 5, 6, 7, and 8 contain a line indicating the changing $B_{0}$ angle of Earth. The $B_{0}$ angles for the two STEREO spacecraft are shown on the outer heliospheric maps in Figure 9 (the upper trace is STEREO-A, the lower trace is STEREO-B). The spacecraft around $\mathrm{L}_{1}$ and the STEREO spacecraft were all located South of the solar equatorial plane for the entire duration of WHI, while Ulysses traveled from $\approx 70^{\circ}$ North heliographic latitude to less than $64^{\circ} \mathrm{N}$. All of the instruments with imaging capabilities (both space- and groundbased) were located near but below the equatorial plane, meaning that the exact North pole of the Sun was not visible in any of the imager data obtained during WHI.

ii) HCS crossings: Maps of both remote-sensing and in-situ data taken near the solar Ecliptic plane (i.e., all but the maps from the Ulysses mission) contain red vertical lines near the $\mathrm{CLON}=239^{\circ}$ and $\mathrm{CLON}=349^{\circ}$ that indicate the location of the crossings of the heliospheric current sheet (HCS) as observed in the ACE data. The region between the lines represents the period where ACE was North of the HCS.

iii) CIR observed by several spacecraft $+\mathrm{ICME}$ at $\mathrm{L}_{1}$ : The maps also contain a blue vertical line around $\mathrm{CLON}=75^{\circ}$, indicating where several ICME properties were observed by ACE (low proton temperature, enhanced helium abundance, and counterstreaming 

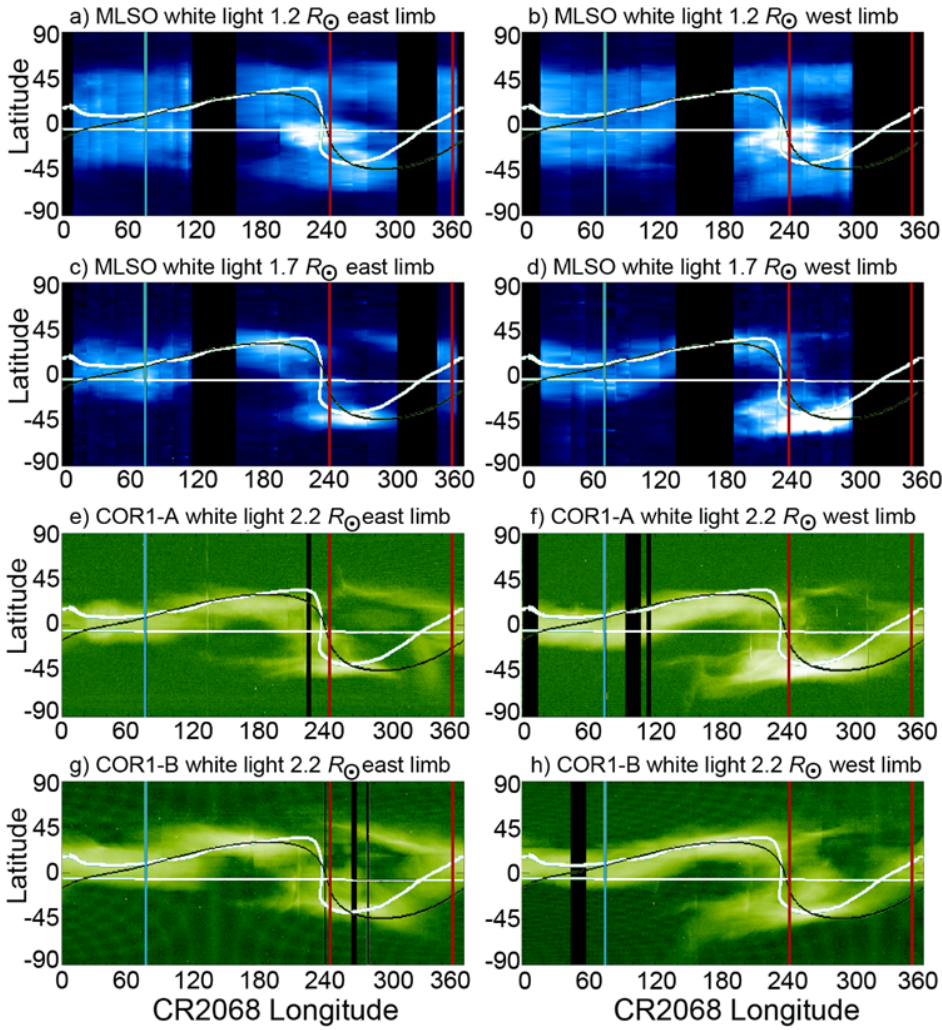

Figure 7 Coronagraph Carrington maps, 1.2-2.2 $R_{\odot}$. (a) Map constructed from MLSO Mk4 white-light data at an altitude of $1.2 R_{\odot}$ above the east limb; (b) MLSO Mk4 map at $1.2 R_{\odot}$ above the west limb; (c) MLSO Mk4 map at $1.7 R_{\odot}$ above the east limb; (d) MLSO Mk4 map at $1.7 R_{\odot}$ above the west limb; (e) STEREO-A/SECCHI/COR1 map at $2.2 R_{\odot}$ above the east limb; (f) STEREO-A/SECCHI/COR1 map at $2.2 R_{\odot}$ above the west limb; (g) STEREO-B/SECCHI/COR1 map at $2.2 R_{\odot}$ above the east limb; (h) STEREO-B/SECCHI/COR 1 map at $2.2 R_{\odot}$ above the west limb.

electrons as identified by J.T. Gosling, personal communication, 2009). The ICME was embedded in a CIR that was observed by both ACE, Wind and STEREO.

iv) Model source surface locations: The maps in Figures 5-8 each contain an overplot of the MAS (Riley et al., 2011) and GONG Potential Field Source Surface (Petrie, Canou, and Amari, 2011) model neutral lines at $R=2.5 R_{\odot}$. The models are described below in Section 3.1.7. The neutral lines are plotted on the Carrington maps at their corresponding heliographic latitudes (white line for MAS, black line for PFSS). In Figure 9, the maps depicting IPS measurements and solar-wind model results contain a trace that indicates the position of the HCS as determined by MAS.

\subsubsection{Residual Subsurface Flows from $7 \mathrm{Mm}$ and $1 \mathrm{Mm}$ below the Photosphere (SOHO/MDI Data)}

The first two maps (labeled $a$ and $b$ ) in Figure 5 show subsurface flow velocities derived from local helioseismological analysis of SOHO/MDI Dopplergram data. The technique, described by Haber et al. (2002), determines horizontal subsurface flows by performing 

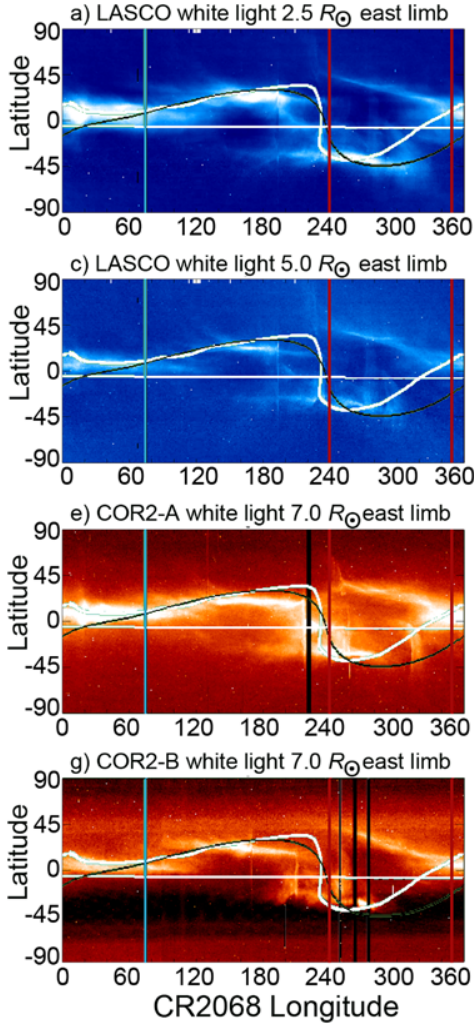

b) LASCO white light $2.5 R_{\odot}$ west limb

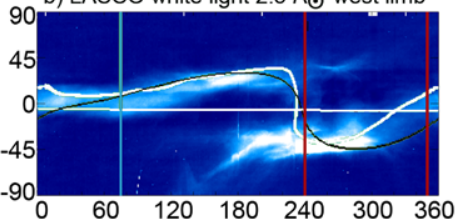

d) LASCO white light $5.0 R_{\odot}$ west limb

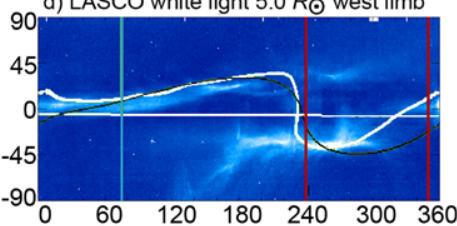

f) COR2-A white light $7.0 R_{\odot}$ west limb

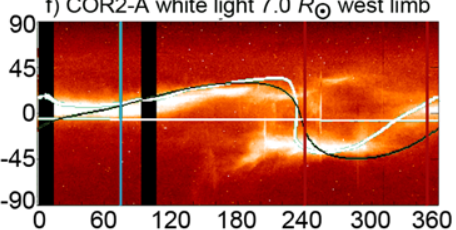

h) COR2-B white light $7.0 R_{\odot}$ west limb

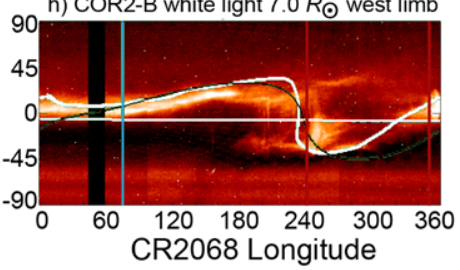

Figure 8 Coronagraph Carrington maps, 2.5 - 7.0 $R_{\odot}$. (a) Map constructed from SOHO/LASCO white light data at an altitude of $2.5 R_{\odot}$ above the east limb; (b) SOHO/LASCO map at $2.5 R_{\odot}$ above the west limb; (c) SOHO/LASCO map at $5.0 R_{\odot}$ above the east limb; (d) SOHO/LASCO map at $5.0 R_{\odot}$ above the west limb; (e) STEREO-A/SECCHI/COR2 map at $7.0 R_{\odot}$ above the east limb; (f) STEREO-A/SECCHI/COR2 map at $7.0 R_{\odot}$ above the west limb; (g) STEREO-B/SECCHI/COR2 map at $7.0 R_{\odot}$ above the east limb; (h) STEREO-B/SECCHI/COR2 map at $7.0 R_{\odot}$ above the west limb.

Optimally Localized Averages (OLA) inversions with the mean zonal and meridional flows removed.

The original flow velocities were determined for a grid of overlapping $15^{\circ} \times 15^{\circ}$ tiles with centers spaced $7.5^{\circ}$ apart. The residual flows were determined by averaging the velocities obtained from all of the available days at each location on the solar disk, and subtracting this average from the individual days. The synoptic maps were then built up by performing a weighted average of the residual flows for each region as it rotated across the solar disk $(\approx$ four to nine days depending on its latitude). The result is a map of the horizontal-flow field as a function of depth in the interior.

The technique has difficulty in resolving flows at higher latitudes, where the solar surface is increasingly tilted away from the observational line of sight. Values can be obtained for latitudes beyond $60^{\circ}$, but the accuracy significantly decreases with latitude; as a result, the map is left blank above $60^{\circ}$ latitude.

Figure $5 \mathrm{a}$ corresponds to a depth of around $7.0 \mathrm{Mm}$, and the second map in Figure 5b corresponds to a depth of $1.0 \mathrm{Mm}$. The magnitude of the flow velocities is represented by the length of the arrows on the map; the arrow in the legend indicates the length of an arrow 

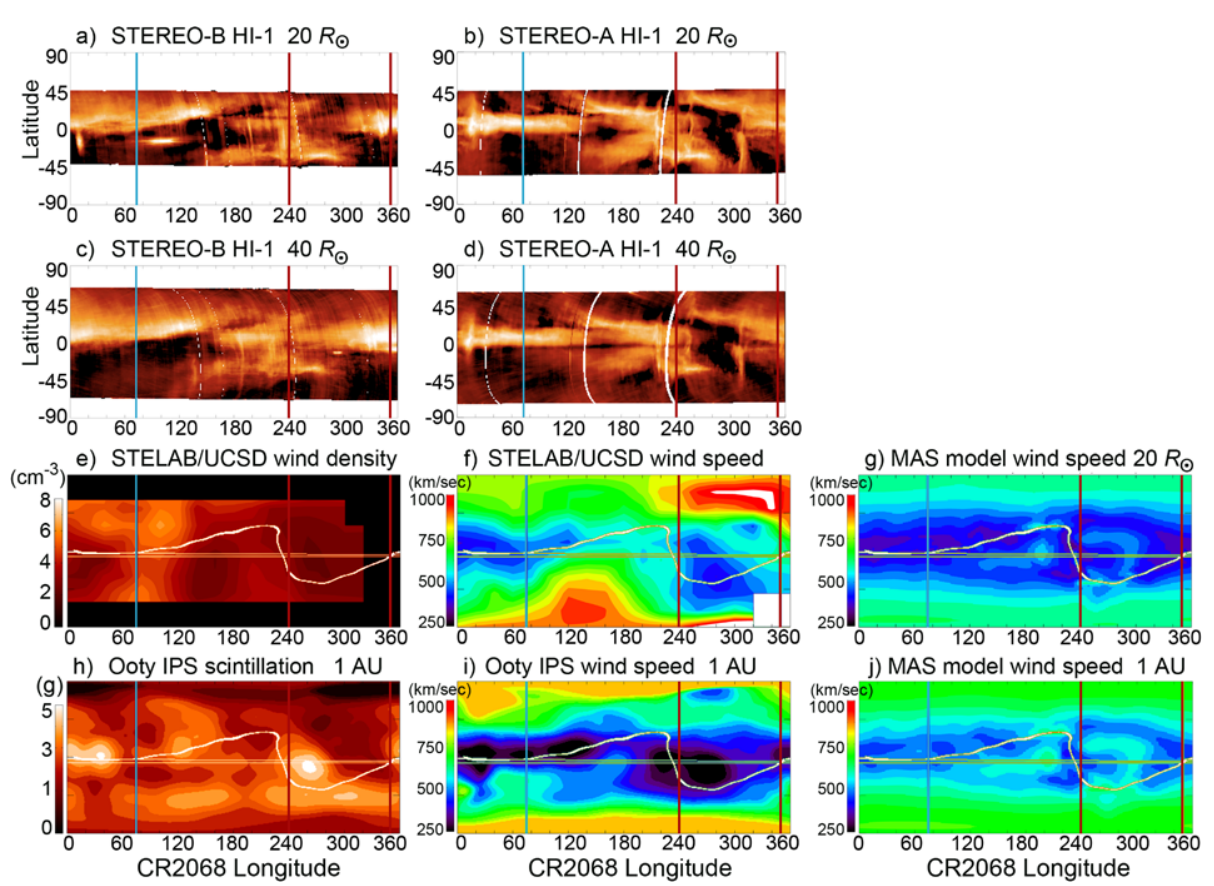

Figure 9 Heliospheric Carrington maps, $15 R_{\odot}-1$ AU. (a) Map constructed from STEREO-B/ SECCHI/HI-1 white-light data at a distance of $20 R_{\odot}$; (b) STEREO-A/SECCHI/HI-1 map at $20 R_{\odot}$; (c) STEREO-B/SECCHI/HI-1 map at $40 R_{\odot}$; (d) STEREO-A/SECCHI/HI-1 map at $40 R_{\odot}$; (e) STELab interplanetary scintillation map of solar-wind density at $15 R_{\odot}$; (f) STELab interplanetary scintillation map of solar-wind speed at $15 R_{\odot}$; (g) MAS model extrapolation of solar-wind speed at $20 R_{\odot}$; (h) Ooty interplanetary scintillation map of solar-wind density at $1 \mathrm{AU}$; (i) Ooty interplanetary scintillation map of solar-wind speed at $1 \mathrm{AU}$; (j) MAS model extrapolation of solar-wind speed at $1 \mathrm{AU}$.

corresponding to $30 \mathrm{~m} \mathrm{~s}^{-1}$. The flow velocities are superposed on magnetogram data (red for positive longitudinal field, green for negative). The subsurface flows at $1 \mathrm{Mm}$ below the photosphere correspond well with the flows $7.0 \mathrm{Mm}$ below the photosphere. The regions with the greatest subsurface flows generally correspond to NOAA Active Regions 10987, 10988, and 10989, located near the solar Equator at Carrington longitudes of $205^{\circ}, 235^{\circ}$, and $260^{\circ}$, respectively, but there are also several other cells present.

Previous studies (e.g. Haber, 2008) have indicated that the strongest and most organized flows are more likely to be near active regions than the quiet Sun. In this case, the flows near AR 10989 were particularly strong, even when compared to the other two active regions. Kosovichev and Duvall (2011) performed a closer examination of the subsurface flow structure under the WHI active regions to a depth of $48 \mathrm{Mm}$. They found that AR 10989 had the strongest shearing flows and was decaying most rapidly, but found no evidence of subsurface linkage between the three regions.

\subsubsection{Longitudinal Magnetogram (Global Oscillation Network Group/GONG)}

Figure $5 \mathrm{c}$ is constructed from GONG longitudinal magnetograms (see Petrie, Canou, and Amari, 2011). As the latitude increases, the accuracy of the magnetograms is affected by the increasingly oblique viewing angle relative to the photospheric surface. The component of the magnetic field along the line-of-sight of the observations decreases, and the cos(latitude) 
projection of the pixels requires increasing interpolation, so values greater than $60^{\circ}$ in latitude are omitted from the map. In addition to the higher latitudes and active regions, there are also many quiet-Sun regions that exhibit a prevailing positive or negative polarity, consistent with the waning phase of the solar cycle.

\subsubsection{Map of Coronal Hole Boundaries and Polarities}

Figure 5d identifies coronal holes and regions of prevailing magnetic polarity using the method described by McIntosh (2003). H $\alpha$ data are examined along with magnetogram and He I $10830 \AA$ from the National Solar Observatory/Kitt Peak to draw the boundaries of magnetic-field regions and coronal holes. The magnetic polarity of the north/south polar coronal holes are identified in red/blue, and equatorial coronal holes assigned colors to match the polarity of the polar holes. The gray/white areas correspond to an overall negative/positive polarity in a given region, with black contours identifying the neutral lines between the opposing field regions. Clusters of mixed polarity near the Equator give way to the polar-crown regions at mid-latitudes and open field lines near the Poles.

\subsubsection{Magnetic Field, Open Field Footpoints, and Projection of Solar Wind (WSA Model)}

Figure 5e shows the distribution of coronal holes as derived by the Wang-Sheeley-Arge model (Arge and Pizzo, 2000) using SOHO/MDI data. Light and dark-gray regions indicate positive and negative longitudinal magnetic flux. Also shown are the field lines that intersect the solar equatorial plane at the model's source surface, traced back to their footpoints at the photosphere (Leamon and McIntosh, 2009). The field lines are calculated by ballistically mapping back along a radial trajectory from the ACE spacecraft at 1 AU to the solar source surface $\left(R=2.5 R_{\odot}\right)$. The field lines are then represented on the map as a straight line between the (latitude, longitude) coordinates of each footpoint and its associated sub-Earth connection longitude in the Ecliptic plane (see Gibson et al. (2011) for a more detailed discussion).

\subsubsection{Magnetic Range of Influence Map}

Leamon and McIntosh (2009) developed a method called the "Magnetic Range of Influence" (MRoI) to describe the connection between the photospheric magnetic field and the solar wind. The MRoI, first proposed by McIntosh, Davey, and Hassler (2006), describes the magnetic environment of a flux element in the photosphere, representing the distance from a SOHO/MDI magnetogram pixel required to balance the integrated magnetic field in the pixel. In Figure 5f, the regions of highest MRoI occur at high latitudes inside coronal holes and at low latitudes in equatorial coronal holes and active regions, and serve as an indicator of the footpoints of the open field lines identified in the PFSS model. The blue regions included in the map label the footpoints of the sub-Earth field lines. The majority of the footpoints connecting to Earth either originate along the boundary of the southern polar coronal hole or in the equatorial coronal holes as identified in the coronal-hole boundary map to the left.

\subsubsection{Map of Coronal Holes Identified in EUV Data (Determined by the de Toma Method)}

Figure 5g shows the coronal-hole boundaries of CR 2068 as determined by the method described by de Toma (2010). This technique uses all four EUV wavelengths available from 
SOHO/EIT and STEREO/SECCHI/EUVI, combining the measurements in each wavelength to take advantage of the different manifestation of coronal holes. In particular, the inclusion of the He II $304 \AA$ (chromospheric-transition region) line helps to better identify boundaries of coronal holes that can be partially obscured by brighter features in optically thin coronal EUV lines (171, 195, and $284 \AA$ ).

\subsubsection{Open-Field Footpoints Determined by PFSS Extrapolation (GONG) and the MAS Model (SOHO/MDI)}

In the maps shown in Figure $5 \mathrm{~h}$ and $\mathrm{i}$, the open field lines as predicted by two different models are presented. The open field lines as determined by the PFSS model (Figure 5h) using GONG data (Petrie, Canou, and Amari, 2011) are similar to those determined by the Wang-Sheeley-Arge method, but show distinct differences from those calculated by the Magnetohydrodynamics Around a Sphere (MAS) model (Riley et al., 2011). The MAS model (Figure 5i) results in a larger volume of open field, including an additional equatorial coronal hole spanning from $15^{\circ}-120^{\circ}$. The manifestation of the surplus coronal hole, nicknamed "Nessie" by the WHI team, depended on the magnetic boundary conditions of the model, particularly the polar fields.

\subsubsection{Radio Emission Map (Nobeyama Radioheliograph)}

Figure $5 \mathrm{j}$ is constructed from $17-\mathrm{GHz}$ observations obtained by the Nobeyama Radioheliograph. The regions of higher emission generally correspond to stronger magnetic field. Note, however, that increased brightness at higher latitudes does not necessarily indicate the location of a polar coronal hole, and that equatorial coronal holes do not appear bright in the $17-\mathrm{GHz}$ images. The cause of the bright polar caps was described by Shibasaki (1998) as a superposition of limb-brightening with intrinsically bright polar features.

\subsubsection{Maps from EUV Imager Data (STEREO/SECCHI and SOHO/EIT)}

The maps in Figure 6 are constructed from EUV images obtained by the SOHO/EIT and STEREO/SECCHI/EUVI instruments. The four wavelengths, $304 \AA, 171 \AA, 195 \AA$, and $284 \AA$, correspond to temperatures of approximately 50000-80000 K (chromospheretransition region), 0.6-0.9 MK (corona), 1.1 - 1.4 MK (corona), and 2.0 MK (corona), respectively. The EUVI images are interpolated over the northern pole, which consists primarily of a coronal hole. The effect of the interpolation is most noticeable in the STEREO-B images, as the spacecraft was located at a lower latitude than STEREO-A at the time of WHI.

At STEREO-B's longitude, CR 2068 began at 6:00 UT on 18 March 2008, while it began at 20:00 UT on 21 March 2008 for STEREO-A. Therefore, an image representing a given Carrington longitude in a STEREO-B map was obtained more than 3.5 days before the corresponding STEREO-A image. There is little variation from one map to the next, for the most part, but some evolution can be spotted at the poleward boundary of the equatorial coronal hole located between $\mathrm{CLON}=110^{\circ}$ and $190^{\circ}$, particularly in the higher-temperature wavelengths (195 $\AA$ and $284 \AA$ ). In the STEREO-B maps, the boundary appears to be weaker and the coronal hole looks more like an equatorward extension of the southern polar hole. Over the next couple of days, the boundary separating the equatorial hole from the Pole appears to solidify, perhaps reflecting the ambiguous results of the different coronal-hole boundary determination techniques shown in Figure 5. 


\subsection{Coronagraph Carrington Maps}

Figures 7 and 8 show the Carrington maps constructed from coronagraph data obtained during WHI. Data from the Mauna Loa Solar Observatory (MLSO) Mk4 coronagraph were used to create the $R=1.2 R_{\odot}$ and $R=1.7 R_{\odot}$ maps. SOHO/LASCO coronagraph data were used for the $R=2.5 R_{\odot}$ maps, COR1 data from STEREO/SECCHI A and B were used for the $R=2.0 R_{\odot}$ maps, and COR2 data were used for the $R=7.0 R_{\odot}$ maps.

To create these maps, an annulus is taken from the coronagraph images at a given altitude. The annulus is divided along the solar apparent meridian, and the corresponding halves become the elements for the east- and west-limb Carrington maps for that altitude.

The $B_{0}$ angle of the coronagraph observations determines the line-of-sight integration path of the data, and therefore there is a slight variation in the structures as they are observed from the different spacecraft position angles. However, the primary difference between the maps is due to two other factors:

i) The variation of the corona in time. The data used to construct the east- and west-limb maps for a given coronagraph are obtained half a Carrington rotation apart, corresponding to nearly two weeks difference in time.

ii) The variation of the heliosphere with altitude. As expected, the streamers become thinner and are located closer to the equatorial plane as the altitude increases.

The white and black lines representing the MAS and PFSS neutral lines at $R=2.5 R_{\odot}$ are shown on all of these maps. A comparison of the source surface with the coronal structure around $2.5 R_{\odot}$ indicates that the models, particularly MAS, reproduce the observations quite well.

\subsection{Heliospheric Carrington Maps}

Figure 9 is a compilation of remote-sensing observations and models representing heliospheric solar-wind structure from $15 R_{\odot}$ out to 1 AU. As explained in Figure 4 , the heliospheric in-situ data and geospace data are "mapped back" to their approximate source Carrington longitude. An application of the back-mapping technique for the 1 AU maps in this section would be unwieldy, because the data are two-dimensional and the solar-wind speed can greatly in latitude as well as longitude. We note that the in-situ observations are offset, on average, by about $45^{\circ}$ from their Parker-spiral footpoint at the Sun. To facilitate comparison with the inner heliospheric maps, we shift the 1 AU maps in Figure 9, as a whole, by $45^{\circ}$.

In order to facilitate comparison with the STEREO spacecraft data, the helio-latitudes of the STEREO spacecraft are superposed on the interplanetary scintillation (IPS) and model solar-wind maps (the upper trace corresponds to STEREO-A, lower trace to STEREO-B). The location of the heliospheric current sheet at $1 \mathrm{AU}$, as determined by the MAS model, is superposed on each of these maps as well.

\subsubsection{Heliospheric Imager Maps (STEREO/SECCHI HI-1)}

In Figure 9a-d, Carrington maps constructed from data obtained by the STEREO/SECCHI/ Heliospheric Imager (HI-1) are presented for comparison with the coronagraph maps in Figures 7 and 8 . The maps represent heights of $20 R_{\odot}$ and $40 R_{\odot}$. The combined field of view of the SECCHI/HI-1 and HI-2 imagers extends from 12 to $215 R_{\odot}$, as the HIs were designed to view CMEs and solar structure during their transit from the Sun to the Earth. On 
STEREO-A the HIs view the east limb of the Sun, and on STEREO-B they view the west limb. These maps help form a bridge between the inner heliospheric observations and the measurements at $1 \mathrm{AU}$, and follow streamers as their latitudinal extent becomes increasingly restricted toward the Equator.

\subsubsection{Interplanetary Scintillation Maps at $15 R_{\odot}$ (STELab/UCSD Tomography IPS)}

Figures $9 \mathrm{e}-\mathrm{f}$ represent the solar-wind density and speed derived through tomographic reconstruction of interplanetary scintillation (IPS) data obtained from the Nagoya University Solar-Terrestrial Environment Laboratory (STELab) IPS array system. The IPS data are obtained through multi-antenna observations of a distant radio source at an observing frequency of $327 \mathrm{MHz}$. Variations in the radio signal, caused by scattering off of small-scale structures in the solar wind, are accumulated as a function of time and elongation angle, and IPS velocity and normalized scintillation-level data are used as input to the tomography to reconstruct the solar-wind velocity and density in the inner heliosphere out to $3 \mathrm{AU}$ (e.g. Jackson and Hick, 2005; Bisi et al., 2009b; and references therein). The 3D tomographic reconstruction involves iterative fits to a solar-wind model that incorporates variations in structure caused by both corotating and outward-flowing structures within the solar wind. (See Bisi et al., 2009a, for a more complete description of how the maps were produced.)

The maps represent the results produced by the tomographic reconstruction at a distance of $15 R_{\odot}$. The velocity reconstructions are complete for CR 2068, but not the density reconstruction, which did not have sufficient numbers of observations to provide complete coverage, particularly at high latitudes (see Bisi et al., 2009a, 2009b). The missing areas of density coverage are left blank in the maps.

Bisi et al. (2009a) performed a thorough comparison between the STELab measurements and solar-wind data from the STEREO/PLASTIC and Wind/SWE instruments. They found that the tomographic reconstructions fit the morphology and magnitude and the in-situ measurements quite well, with the weakest fit being near a high-density feature measured at STEREO-A.

\subsubsection{Interplanetary Scintillation Maps at 1 AU (Ooty Radio Telescope)}

Figures $9 \mathrm{~h}-\mathrm{i}$ show solar-wind speed and normalized scintillation level ( $g$-level) derived from observations of IPS using the Ootacamund (Ooty) Radio Telescope. The velocities are reconstructed tomographically with a method similar to the one used to produce the STELab maps. These maps represent the solar wind structure at 1 AU, and Manoharan (2010) discuss the differences between the measurements taken this solar cycle $v s$. the previous solar cycle. In particular, the low-speed region along the equatorial belt is considerably wider than that of the previous cycle, consistent with the observations of weaker polar magnetic fields. As mentioned previously, these maps and the following map are shifted by $45^{\circ}$ in longitude, to offset the average position of the Parker-spiral footpoints.

\subsubsection{Solar Wind Speed and Heliospheric Current Sheet Derived by the MAS Model}

The maps in Figure $9 \mathrm{~g}$ and $\mathrm{j}$ show the solar-wind speed at $20 R_{\odot}$ and $1 \mathrm{AU}$ as derived by the MAS model. These maps are the solar-wind counterpart to the MAS open-footpoint map in Figure 5, and are presented for comparison to the IPS measurements, particularly at midto-high latitudes (as was the case with a similar comparison of CR 2029 in the declining phase of the solar cycle; Bisi et al., 2010b). The reasons for such differences are still a topic 


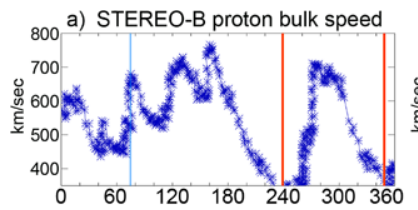

b) Wind proton bulk speed

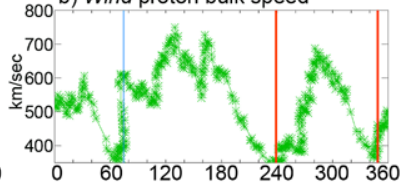

e) Wind proton density

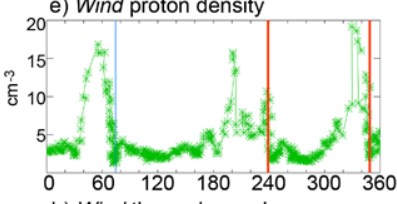

h) Wind thermal speed
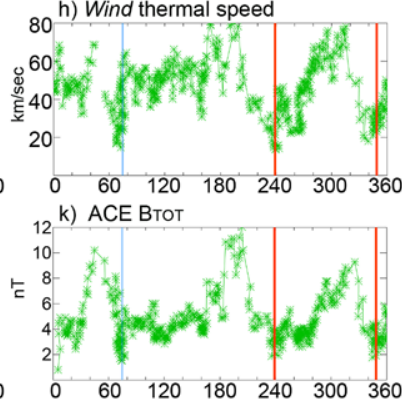

0.5 n) ACE $07 / 06$ composition ratio

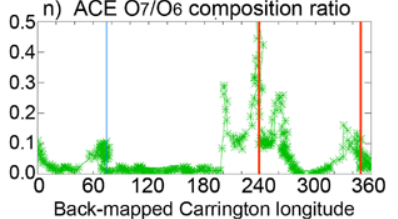

c) STEREO-A proton bulk speed
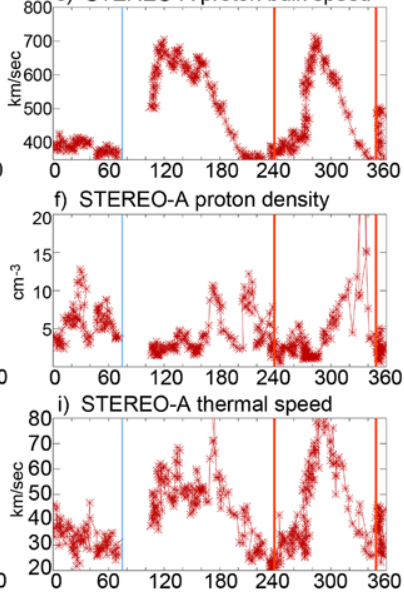

10 I) ACE GSM Bz

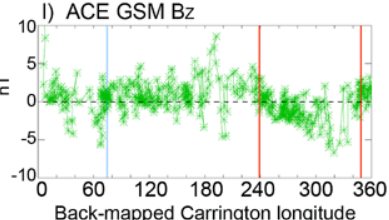

Back-mapped Carrington longitude

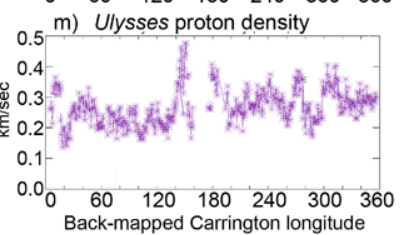

Figure 10 Carrington coordinate plots of in-situ solar-wind data, back-mapped according to the technique shown in Figure 4. (a) STEREO-B/PLASTIC proton bulk speed; (b) Wind/SWE proton bulk speed; (c) STEREO-A/PLASTIC proton bulk speed; (d) STEREO-B/PLASTIC proton density; (e) Wind/SWE proton density; (f) STEREO-A/PLASTIC proton density; (g) STEREO-B/PLASTIC thermal speed; (h) Wind/SWE thermal speed; (i) STEREO-A/PLASTIC thermal speed; (j) Ulysses/SWOOPS proton bulk speed; (k) ACE/MAG magnetic-field intensity; (1) ACE/MAG $B_{Z}$; (m) Ulysses/SWOOPS proton density; (n) ACE/SWICS $\mathrm{O}^{+7} / \mathrm{O}^{+6}$ composition ratio.

of investigation. A comparison of the MAS model with STEREO in-situ measurements at 1 AU (Riley et al., 2011) revealed that the model's range of velocities matched well with the observations at a $1 \mathrm{AU}$ distance from the Sun near the Ecliptic plane.

\subsection{In-situ Solar-Wind Data}

Figure 10 shows in-situ measurements mapped back in the manner illustrated in Figure 4. The bulk proton speed, density, and thermal speed are all shown for three different heliolongitudes (STEREO-B, Wind and STEREO-A). The ACE $\mathrm{O}^{+7} / \mathrm{O}^{+6}$ composition ratio, total magnitude of the interplanetary magnetic field (IMF), and $B_{Z}$ in Geocentric Solar Magnetospheric (GSM) coordinates are all shown.

There are clear similarities between the STEREO-B, Wind, and STEREO-A proton bulk speed measurements, but there is also some variation in structure between the maps. (Note: There was an outage of the STEREO-A measurements during the dates corresponding to $\mathrm{CLON}=75^{\circ}$ to $105^{\circ}$.) At the longitude of STEREO-B, CR 2068 started 43 hours earlier 
than at Earth, and STEREO-A started 43 hours later. Additionally, the heliographic latitudes were different. STEREO-B ranged from $B_{0}=-7.1^{\circ}$ to $-7.3^{\circ}$, STEREO-A ranged from $B_{0}=-5.6^{\circ}$ to $-2.7^{\circ}$, and Earth was in between, slightly closer to the STEREO-B latitudes. This means that slowly evolving structures observed at $\mathrm{L}_{1}$ would be closer in proximity, and presumably morphology, to the STEREO-B observations than the STEREO-A observations. Upon inspection, this appears to be true for the bulk-speed and thermal-speed measurements, but not as much for the density. The CIR structure that appears in both the Wind and ACE data from CLON $=35^{\circ}$ to $75^{\circ}$ is much smaller at both STEREO locations, at least in part due to the ICME accompanying the observations at $\mathrm{L}_{1}$.

We must place limitations on any conclusions that we draw from a simple comparison between the lower-latitude data to the Ulysses measurements. As mentioned earlier, the lower-latitude spacecraft spend most of WHI below the HCS, except for the period between $\mathrm{CLON}=239^{\circ}$ and $349^{\circ}$. The solar-wind speed and density measured by Ulysses during WHI exhibits much less variation than the measurements near the Ecliptic plane, consistent with a solar-wind source in the northern polar coronal hole. The high-speed wind observed by ACE, STEREO, and Wind likely originates in an equatorial coronal hole and not the northern pole, so the Ulysses data may have little overlap with the other in-situ observations.

However, the Ulysses measurements serve as an extremely important constraint for the heliospheric models, as the solar poles have played a major role in establishing our "unusual" solar-minimum corona. As expected, the magnetic-field magnitude shows the greatest increases near the heliospheric current sheet (HCS) crossings and high-speed stream (HSS) interfaces, and the composition ratio shows a great deal of variability near the heliospheric current sheet, but not as much at the boundary of the HSS at CLON $=75^{\circ}$. Fisk and Zhao (2009) and Zhao and Fisk (2011) investigated these solar-wind properties and found that they are consistent with our understanding of the heliosphere; i.e. the mass flux, temperature, and pressure relationships of the fast and slow solar wind are unchanged for this minimum.

\subsection{Geospace Data}

The geospace data in Figure 11 are presented in the same manner as the in-situ solar-wind data, as a function of Carrington longitude instead of time. As is shown in Figure 4, in these plots time goes from right to left on the $x$-axis. The vertical lines marking the HCS crossings and the ICME/CIR are also superposed on these plots.

\subsubsection{Radiation Belt Electron Flux (NOAA/GOES)}

Figure 11a shows the $>2 \mathrm{MeV}$ electron flux in the outer radiation belt as measured by the NOAA/GOES satellites. As described by Gibson et al. (2009), the peak radiation-belt values were elevated by more than three times over those observed during the Whole Sun Month (1996) campaign from the previous solar minimum. The radiation-belt electron flux later declined to its lowest recorded levels in late 2009. The GOES $>2 \mathrm{MeV}$ electron-number fluxes correlate best with the solar-wind speed (e.g. Baker et al., 1990; Emery et al., 2011), although there is always a drop-out in the electron flux at the leading edge of an HSS because the radiation belt is pushed Earthwards by the increased solar-wind dynamic pressure.

\subsubsection{Auroral Electron Power (DMSP)}

Figure 11b shows auroral electron power derived from hourly averages of intercalibrated measurements made by the NOAA Polar-orbiting Operational Environmental Satellite 

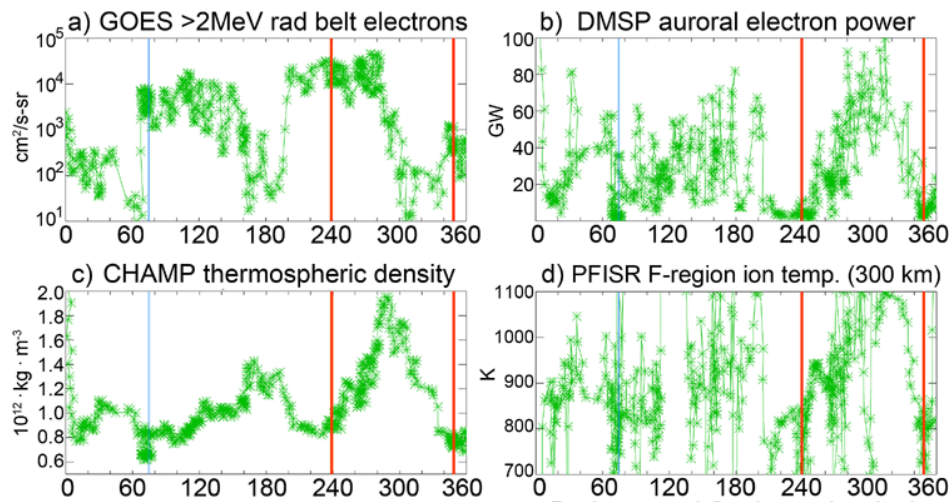

d) PFISR F-region ion temp. $(300 \mathrm{~km})$

e) TIMED/SABER NO cooling flux
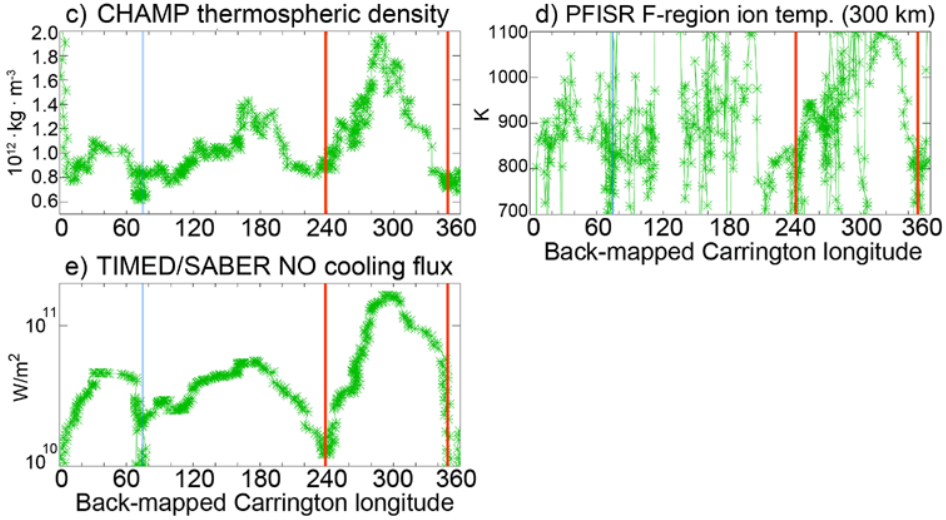

Figure 11 Carrington coordinate plots of geospace and upper-atmospheric data, back-mapped according to the technique shown in Figure 4. (a) GOES radiation-belt electron flux; (b) DMSP auroral electron power; (c) CHAMP thermospheric densities; (d) PFISR F-region ion temperature; (e) TIMED/SABER NO cooling flux.

(POES) and US Air Force Defense Meteorological Satellite Program (DMSP) satellites. Gibson et al. (2009) also found a correlation between the solar-wind speed periodicity and magnitude and the auroral electron power. The auroral electron-power input was largest for the first HSS when the Earth was above the HCS and IMF $B_{Z}$ was mostly negative. However, the auroral input was still significant for the middle HSS during mostly positive IMF $B_{Z}$ conditions due to the Alfvénic fluctuations at the magnetopause during HSSs (Echer $e t$ al., 2011). This is consistent with the correlations found by Emery et al. (2009) of the auroral power with solar-wind electric field during $B_{Z}$-negative or positive conditions, where the correlations were highest during HSSs for $B_{Z}$-negative conditions.

\subsubsection{Thermospheric Density (CHAMP)}

Figure 11c, Challenging Minisatellite Payload (CHAMP) accelerometer data are used to derive thermospheric densities (see Lei et al., 2011). The measurements at satellite orbits are normalized to an altitude of $400 \mathrm{~km}$ using the NRLMSISE00 empirical model (Picone $e t$ al., 2002). The CHAMP density shows clear responses to the three CIR/HSS events during the Carrington rotation. Model calculations by Solomon et al. $(2010,2011)$ allowed a close examination of the relationship between the solar EUV irradiance and the unusually low thermospheric densities observed near the recent solar minimum. The low variation in solar EUV flux during WHI provides a unique opportunity to isolate the effect of solar-wind drivers of thermospheric variation. As revealed by Lei et al. (2011), the geomagnetic activity associated with the HSSs had a significant impact on the thermosphere. 


\subsubsection{F-Region Ion Temperatures (PFISR)}

Figure 11d shows F-region ion temperature at $300 \mathrm{~km}$, constructed from measurements from the Poker Flat Advanced Modular Incoherent Scatter Radar (PFISR). The data show a nearperfect correspondence with the auroral-electron power, indicating a common driver. Sojka et al. (2011) examined fifty CIRs from March 2007 - February 2008 as part of an International Polar Year (IPY) campaign, and found that each CIR had an observable response in the PFISR data that lasted several days.

\subsubsection{Nitric Oxide Cooling Flux (TIMED/SABER)}

Figure 11f shows TIMED/SABER measurements of infrared radiative cooling by nitric oxide in the Earth's thermosphere. Not surprisingly, the cooling flux shows a strong correlation to the thermospheric-density measurements from CHAMP, serving as more evidence of the geospace response to the CIRs. Mlynczak et al. (2010) found that, as opposed to $\mathrm{CO}_{2}$, the cooling rates and radiative fluxes from NO are largest at high latitudes and polar regions, and they exhibit substantially higher solar-cycle variability.

\section{Conclusions}

WHI began as an extension of the "Whole Sun Month" concept, building on the successful program from the previous solar minimum by adding modern modeling techniques, increased 3D heliospheric coverage, and extending the observations to include the impact on geospace. A comprehensive suite of models and observations were formulated to allow the WHI team to thoroughly address the three primary science questions described in Section 2.1 of this article.

Although the "unusual" solar minimum between Solar Cycles 23 and 24 occurred much later than originally anticipated, it provided an excellent opportunity to learn more about the nature of not only the interconnected heliophysical system, but the nature of solar minimum in general: When your observations give you lemons, study lemonade.

WHI also provided the opportunity to study the cause and effect of phenomena that are difficult to isolate during times of higher activity. Throughout WHI, geospace was "ringing" with the impact of recurrent high-speed streams (HSS). The processes governing these interactions are always present, but during solar maximum a steady stream of CMEs, flares, and EUV irradiance variations are added to the mix.

Several years of concerted effort have produced breakthroughs and insights. Still, it is clear that the picture is far from complete. While WHI shed a spotlight on many fundamental heliophysical processes, many questions remain. A major limitation of our Carrington-map approach is that the montage does not adequately represent variations on smaller timescales. Despite the coarse granularity of the Carrington maps and plots, a picture starts to emerge, but much of the important physics lies in boundaries and interactions that are more intricate or subtle. It may be clear in the overview plots that some aspects are interconnected, but the "how" and "why" are usually hidden in the details. Therefore, we now take the opportunity to highlight some of the more focused collaborative studies undertaken by the WHI team.

\subsection{Completing the Montage}

The data and models presented in this article provide only a brief glimpse of the heliosphere's slow descent to solar minimum. As seen in the solar Carrington maps presented 
in Section 3, the WHI Sun was dominated by several traditionally "non-solar-minimum" features. Most of the global coronal-field structure around CR 2068/WHI consisted of quiet Sun, and nearly all significant magnetic activity was crowded between CLON $=180^{\circ}$ and $270^{\circ}$. This lopsided activity distribution, combined with relatively weak polar fields, resulted in a global coronal-field structure that was far from the nearly axisymmetric, dipolar configuration expected during solar minimum. There was a large warp in the streamer belt centered at around $239^{\circ}$ of Carrington longitude, and much of the flux connecting to the Ecliptic plane originated from low-latitude coronal holes, an unusual state of affairs for a near-minimum corona. These were responsible for, and increased prevalence of, high-speed streams and CIRs, and the majority of the geospace impacts (Gibson et al., 2009, 2011; Emery et al., 2011).

Several WHI studies focused on the solar magnetic origin of these CIRs (e.g. Riley, Linker, and Mikić, 2010; Petrie, Canou, and Amari, 2011; Riley et al., 2011), as well as their extent and morphology. de Toma (2010) examined the polar magnetic fields of the Sun, and discussed the role of low-latitude coronal holes and high-speed streams in determining heliospheric structure, as did Bisi et al. (2010a), Maris and Maris (2010), and Jian, Russell, and Luhmann (2011). Others have studied and documented their connection to geospace (see below) as well as other planetary systems (Jackman and Arridge, 2011).

As mentioned in Section 2, solar-irradiance studies were also a fundamental part of WHI. The direct energy input to geospace, and the question of the Earth's energy budget overall, require a clear understanding and characterization of solar irradiance from EUV to infrared wavelengths. The effort described by Chamberlin et al. (2009) and Woods et al. (2009) resulted in the most comprehensive Solar Irradiance Reference Spectra (SIRS) that had ever been produced. White (2011) used measurements from several sources to characterize the Sun's radiative output, and found that although the values were already extremely low at the time of CR 2068/WHI, the minimum of each of the time series occurred in the last quarter of 2008, which was consistent with the date of the sunspot minimum. EUV irradiance modeling by Haberreiter (2011) and Solomon et al. (2010, 2011) allowed a closer examination of the causes of irradiance variations near solar minimum. Additionally, thermospheric modeling done by Solomon et al. $(2010,2011)$ established a clear link between the long lull in solar activity and the unusually low density and temperature of the thermosphere.

The modeling efforts, however, could not provide sufficient explanation for the $10 \%$ lower EUV irradiance in 2008 than in 2006. The abundance of coronal holes at lower latitudes can explain about $5 \%$ of the lower 2008 irradiance from examining TIMED/SEE and SOHO/EIT data. Consequently, there is another $5 \%$ of unexplained source of lower EUV irradiance, possibly caused by the quiet-Sun radiance being slightly lower due to reduced solar magnetic fields in 2008 than in 1996. From an irradiance standpoint, the dominant solar-surface features, namely the active regions and active network, are not very different in 2008 from those in 1996 and thus cannot explain the deficit. It remains an open question.

\subsection{A Sun with Two Personalities}

Another important and seemingly paradoxical question relates to a different aspect of solar variability. Webb et al. (2011) identified all of the CMEs that occurred during Carrington rotations 2067, 2068, and 2069, and found that $73 \%$ of the CMEs with identifiable sources occurred during CR 2068. In addition to the prevalence of high-speed streams, the WHI Sun also exhibited an unexpectedly high level of solar activity. The most energetic activity was confined to a closely spaced cluster of three active regions (NOAA ARs 10987, 10988 and 10989) that, despite their close proximity, differed appreciably in structure and behavior. 
Petrie, Canou, and Amari (2011) calculated PFSS models for CR 2068 based on GONG line-of-sight magnetograms, and Nonlinear Force-Free Field (NLFFF) models based on SOLIS vector-magnetogram data for the these three active regions. The PFSS model for CR 2068 showed that the three regions, which were spaced about $30^{\circ}$ apart in longitude and about $10^{\circ}$ in latitude, were highly interconnected. ARs 10987 and 10988 were located mostly within the helmet-streamer belt, whereas most of AR 10989 lay outside the streamer belt among open fields. The NLFFF models enabled a closer analysis of energy balance, but they found that the coronal AR magnetic fields did not indicate levels of free magnetic energy or helicity normally associated with flaring active regions. However, AR 10987 had a coherent S-shaped or sigmoidal structure connecting its two sunspots, which has been shown to be a strong eruption precursor (Canfield, Hudson, and Pevtsov, 2000).

Welsch, Christe, and McTiernan (2011) analyzed several predictors of flare activity based on the photospheric line-of-sight magnetic field, and their results indicated that AR 10988 should have been the most active region of the three and AR 10989 the least. Consistent with the findings from the NLFFF models of Petrie, Canou, and Amari (2011), they found that, of the three regions, AR 10989 had the lowest free magnetic energy and relative magnetic helicity. Webb et al. (2011) showed, however, that it was AR 10989, and not AR 10987, that was the most CME- and flare-productive of the three regions.

Nitta (2011) noted that two of the five fastest ( $>900 \mathrm{~km} \mathrm{~s}^{-1}$ ) CMEs in 2007-2009 occurred during WHI, one from AR 10987 and one from AR 10989. He also looked for correlations with several activity precursors (microflares, swelling at the streamer base, and filament evolution), but found no obvious reason why ARs 10987 and 10989 would produce extremely fast CMEs.

Each of the aforementioned authors began to seek possible reasons why AR 10989 was unexpectedly active. The answer appears to come from two directions: above and below. Evidence for a potential "below" connection was seen in the subsurface Carrington maps in Figure 5, which showed higher flow structure beneath the active-region complex. An examination of the subsurface flows, particularly the strong neighboring vortical flows of opposite sign around AR 10989, led Kosovichev and Duvall (2011) and Webb et al. (2011) to suggest a connection between the interior and the unusually high activity from AR 10989. As with flare forecasts, it is becoming increasingly clear that time-distance helioseismology is a powerful tool in predicting potential CME activity as well.

Petrie, Canou, and Amari (2011) and Nitta (2011) found compelling evidence that the connectivity of the active regions to the rest of the corona was also a major factor, particularly with regards to connection or access to open field lines. AR 10988 was confined under large-scale closed field, while AR 10989 was situated among open fields. Even if the active region itself contains little free energy or eruption potential, its interaction with and connection to the large-scale corona cannot be neglected. By looking beyond the active regions in question, particularly above and below them, the investigators helped us understand why our quiet Sun was not so quiet.

\subsection{The Response at Geospace}

With the unusually high rate of solar magnetic activity during WHI, one would expect some dramatic effects at Earth. However, the "geospace story" was all about the high-speed streams, with very little contribution from ICME-associated activity. Cremades, Mandrini, and Dasso (2011) and Lepping et al. (2010) provided important clues as to why this would be so. Cremades, Mandrini, and Dasso (2011) performed detailed analyses of the many interplanetary CME structures and determined their origin and connection to the solar-disk 
activity. They identified two key, related factors that could explain the small impact of CMEs on geospace: $i$ ) The weak polar fields of the Sun resulted in a significant decrease in the deflection of CMEs toward the Ecliptic plane, and ii) the highly warped and tilted current sheet meant that Earth spent more time outside the central band of slow-speed wind. Lepping et al. (2010) investigated the properties of the ICMEs with magnetic-cloud morphology, which are particularly important for geospace consequences because they frequently consist of a strong, steady, and persistent magnetic field. They found that relative to the past solar minimum, the clouds were fewer (62\% decrease in frequency), weaker (33\% decrease in axial magnetic-field strength), and smaller (27\% decrease in average duration). As explained by Lepping et al. (2010), the change in average properties of magnetic clouds can be viewed as being consistent with the observations of a weaker overall magnetic field of the Sun.

Interestingly, many of the factors that reduced the CME impacts on geospace (weak polar fields, warped current sheet, equatorial coronal holes) were responsible for the dominance of high-speed streams and their profound geospace impact during WHI. The geoefficiency of the solar wind at Earth is primarily controlled by the IMF $B_{Z}$ component, where the largest geoefficiencies correspond to negative GSM $B_{Z}$ periods. As explained by Echer, Tsurutani, and Gonzalez (2011), the location of the Earth above or below the HCS can lead to enhanced or reduced GSM $B_{Z}$ from the Russell and McPherron (1973) effect. The Earth's spin axis is tilted in the negative $y$-axis direction during Spring, so if $B_{X}$ is positive (toward, and $B_{Y}$ is negative), then GSM $B_{Z}$ will be more negative. This is the case between CLON = $239^{\circ}-349^{\circ}$ for the first HSS when the Earth is above the HCS. For the second, longer HSS, the Earth is below the HCS and GSM $B_{Z}$ is more positive, as is seen in Figure 10. Echer, Tsurutani, and Gonzalez (2011) showed that the geomagnetic indices of the auroral electroject (AE) and the disturbed (Dst) equatorial current were larger for the first HSS than for the second. Wang et al. (2011) showed that Kp and the ionospheric-storm responses were larger for the first HSS than for the second. Indeed, increased responses for negative IMF GSM $B_{Z}$ can be seen in the larger responses in the geospace parameters in Figure 11 between the red vertical lines marking the HCS crossings.

As discussed in Section 3.5, there was a clear correspondence between the HSSs and several additional indicators of geomagnetic activity. Lei et al. (2011) looked at the periodicity of the IMF ( $B_{\text {TOT }}, B_{X}$, and $\left.B_{Z}\right)$ in 2008. $B_{X}$ only had peaks at 13.5 and 27 days with a minor peak at seven days, while the strongest peak in $B_{\text {TOT }}$ was at nine days. The ionosphere and thermosphere respond most strongly to negative IMF $B_{Z}$, where the periodicity in $B_{Z}$ was mostly around 27, and 13.5 days, with a significant peak at seven days.

Wang et al. (2011) found that the periodicities in $B_{\mathrm{TOT}}$ and $B_{Z}$ for three Carrington rotations centered around CR 2068/WHI were similar to those for the entire year. Most of the parameters in Figure 11 share the nine-day periodicity of the solar wind, including the peak electron density in the F region ( $\mathrm{NmF} 2)$ at all latitudes studied by Wang et al. (2011). In addition, ionosonde stations near the geomagnetic equator also exhibited an equally strong seven-day periodicity, which was similar to the $B_{Z}$ seven-day periodicity.

Emmert, Lean, and Picone (2010) showed that the neutral density at $400 \mathrm{~km}$ during the WHI solar minimum was $28 \%$ lower than the previous solar minimum in 1996. Solomon et al. (2010) suggested that this was consistent with the observed EUV irradiance decrease, including a 15\% decrease from 1996 to 2009 in the He $304 \AA$ emission observed by the SOHO Solar Extreme-ultraviolet Monitor (SEM). Araujo-Pradere et al. (2011) looked at NmF2 and the vertical total electron content (vTEC) at several mid-latitude locations for summer and winter months between 1996-1997 and 2007-2009 to see if there was a similar decrease between the WSM solar minimum (1996) and WHI solar minimum. The results were inconclusive for NmF2, but vTEC values did show a decrease of the total electron content in 
the ionosphere and plasmasphere from the ground to $20000 \mathrm{~km}$ (the altitude of all global positioning satellites) between 1996 and 2008, especially in early evening hours. They also found a decrease in vTEC between $2006-2007$ and 2008-2009.

The CR 2068/WHI 1 period in geospace was more active than the subsequent WHI 2 and 3 periods. Most geospace quantities, such as the radiation-belt electron-number fluxes, the auroral fluxes, and the geomagnetic Kp and Ap indices, reached their minimum values in late 2009 (Emery et al., 2011) reaching historically low values (Echer, Tsurutani, and Gonzalez, 2011). But during WHI 1, these and other geospace parameters in Figure 11 were found to "ring" with the periodicities in the solar-wind structure, consistent with the (still) declining phase of the solar cycle (Gibson et al. 2009, 2011). CME activity at the Sun was anomalously high, but it had little or no impact on geospace. Several apparent paradoxes presented themselves, but with close examination a more physical understanding became available.

\subsection{An Unusual Solar Minimum?}

Many of the topics addressed by WHI researchers led to even more open questions about not only this most recent solar minimum, but all solar minima in general. Properties of many phenomena and data exhibited distinct differences from the previous solar minima. People began looking for systematic explanations and wider connections, so the question inevitably became "Was this solar minimum unusual?" As the evidence accumulated, it became clear that although this past minimum was indeed unusual, most of the conclusions were based on measurements and modeling techniques that have only been available for a few solar cycles. The question then became "Was this solar minimum unusually unusual?"

In addition to many studies that have been published over the past three years, several articles in this Topical Issue continue to shed light on this subject. It appears that the answer to that last question depends on one's definition of "unusual." Regardless, the extended decay of Solar Cycle 23, and the later-than-anticipated arrival of Cycle 24, provided an ideal opportunity to study the interconnected 3D heliophysical system, and brought us several steps closer to understanding the fundamental processes governing these interactions.

Acknowledgements The authors extend their sincere gratitude to the following for useful discussions and/or assistance in providing data/models/results used in this publication: A. Balogh, Ulysses/VHM Principal Investigator, Imperial College of Science, Technology and Medicine, London (UK); J.M. Clover and P.P. Hick, University of California, San Diego (USA); J. Davies, Rutherford Appleton Laboratory (UK); Andrew Davis, ACE Science Center at Caltech (USA); R.A. Fallows, Aberystwyth University, Wales (UK); R. Fuller-Rowell, T. Onsager, and A. Reinard, NOAA/SWPC, Boulder (USA); Nat Gopalswamy, NASA/GSFC, Greenbelt MD (USA); J.T. Gosling, University of Colorado, Boulder (USA); J.U. Kozyra, University of Michigan, Ann Arbor (USA); M. Lancaster and C. Tranquille, Ulysses Data System, ESA/ESTEC (Netherlands); J.G. Luhmann, STEREO/IMPACT Principal Investigator, UC Berkeley/SSL (USA); D.J. McComas, Ulysses/SWOOPS Principal Investigator, Southwest Research Institute (USA); S.W. McIntosh and S. Solomon, UCAR/HAO, Boulder (USA); M. Mlynczak, NASA/Langley Research Center and the TIMED/SABER team; J. Sojka, Utah State University (USA); A. Szabo, Wind Project Scientist, NASA/GSFC (USA). The electron auroral power data were supplied by the Coupling, Energetics and Dynamics of Atmospheric Regions (CEDAR) Database, which is supported by the National Science Foundation.

B.J. Thompson also thanks A.C. Rager for her assistance in editing this article.

The authors would also like to thank David Hathaway of NASA/MSFC for his solar-cycle forecast and sunspot number data at http://solarscience.msfc.nasa.gov/SunspotCycle.shtml

Information on the NOAA/NASA/ISES Solar Cycle 24 Prediction Panel (D.A. Biesecker, Chair) can be found at http://www.swpc.noaa.gov/SolarCycle/SC24/index.html

The National Center for Atmospheric Research is supported through the National Science Foundation. FISR is operated by SRI International under NSF cooperative agreement ATM-0608577. L. Qian's effort was supported by NASA Heliophysics Guest Investigator Grant \#NASA NNH08ZDA001N-HGI. 
Open Access This article is distributed under the terms of the Creative Commons Attribution Noncommercial License which permits any noncommercial use, distribution, and reproduction in any medium, provided the original author(s) and source are credited.

\section{References}

Arge, C.N., Pizzo, V.J.: 2000, Improvement in the prediction of solar wind conditions using near-real time solar magnetic field updates. J. Geophys. Res. 105, 10465. doi:10.1029/1999JA900262.

Altrock, R.C.: 2011, Coronal Fe XIV emission during the whole heliosphere interval campaign. Solar Phys. doi:10.1007/s11207-011-9714-9.

Araujo-Pradere, E.A., Redmon, R., Fedrizzi, M., Viereck, R., Fuller-Rowell, T.J.: 2011, Some characteristics of the ionospheric behavior during the solar cycle 23-24 minimum. Solar Phys. doi:10.1007/ s11207-011-9728-3.

Aschwanden, M.J.: 2011a, The state of self-organized criticality of the Sun during the last three solar cycles. I. Observations. Solar Phys. doi:10.1007/s11207-011-9755-0.

Aschwanden, M.J.: 2011b, The state of self-organized criticality of the Sun during the last three solar cycles. II. Theoretical modeling. Solar Phys. doi:10.1007/s11207-011-9835-1.

Baker, D.N., McPherron, R.L., Cayton, T.E., Klebesadel, R.W.: 1990, Linear prediction filter analysis of relativistic electron properties at 6.6 Re. J. Geophys. Res. 95(A9), 15133-15140. doi:10.1029/ JA095iA09p15133.

Biesecker, D.A., Thompson, B.J., Gibson, S.E., Alexander, D., Fludra, A., Gopalswamy, N., Hoeksema, J.T., Lecinski, A., Strachan, L.: 1999, Synoptic Sun during the first whole Sun month campaign: August 10 to September 8, 1996. J. Geophys. Res. 104, 9679. doi:10.1029/1998JA900056.

Bisi, M.M., Jackson, B.V., Buffington, A., Clover, J.M., Hick, P.P., Tokumaru, M.: 2009a, Lowresolution STELab IPS 3D reconstructions of the whole heliosphere interval and comparison with in-ecliptic solar wind measurements from STEREO and Wind instrumentation. Solar Phys. 256, 201. doi:10.1007/s11207-009-9350-9.

Bisi, M.M., Jackson, B.V., Hick, P.P., Buffington, A., Clover, J.M.: 2009b, Coronal mass ejection reconstructions from interplanetary scintillation data using a kinematic model: a brief review. In: Duldig, M. (ed.) Adv. Geosciences 14, World Scientific, Singapore, 161. http://adsabs. harvard.edu/abs/2009aogs...14..161B.

Bisi, M.M., Jackson, B.V., Clover, J.M., Hick, P.P., Buffington, A.: 2010a, A summary of 3-D reconstructions of the whole heliosphere interval and comparison with in-ecliptic solar wind measurements from STEREO, ACE, and wind instrumentation. In: Corbett, I.F. (ed.) Whole Heliosphere Interval: Overview of JD16, Highlights of Astronomy 15, Cambridge Univ. Press, Cambridge, 480. doi:10.1017/S1743921310010331.

Bisi, M.M., Breen, A.R., Jackson, B.V., Fallows, R.A., Walsh, A.P., Mikić, Z., Riley, P., Owen, C.J., Gonzalez-Esparza, A., Aguilar-Rodriguez, E., Morgan, H., Jensen, E.A., Wood, A.G., Owens, M.J., Tokumaru, M., Manoharan, P.K., Chashei, I.V., Giunta, A.S., Linker, J.A., Shishov, V.I., Tyul'bashev, S.A., Agalya, G., Glubokova, S.K., Hamilton, M.S., Fujiki, K., Hick, P.P., Clover, J.M., Pintér, B.: 2010b, Remote sensing of the inner heliosphere. Solar Phys. 265, 49. doi:10.1007/s11207-010-9602-8.

Canfield, R.C., Hudson, H.S., Pevtsov, A.A.: 2000, Sigmoids as precursors of solar eruptions. IEEE Trans. Plasma Sci. 28, 1786. doi:10.1109/27.902208.

Chamberlin, P.C., Woods, T.N., Crotser, D.A., Eparvier, F.G., Hock, R.A., Woodraska, D.L.: 2009, Solar cycle minimum measurements of the solar extreme ultraviolet spectral irradiance on 14 April 2008. Geophys. Res. Lett. 36, L05102. doi:10.1029/2008GL037145.

Cliver, E.W., Ling, A.G.: 2011, The floor in the solar wind magnetic field revisited. Solar Phys. doi:10.1007/s11207-010-9657-6.

Cremades, H., Mandrini, C.H., Dasso, S.: 2011, Coronal transient events during two solar minima: Their solar source regions and interplanetary counterparts. Solar Phys. doi:10.1007/s11207-011-9769-7.

de Toma, G.: 2010, Evolution of coronal holes and implications for high-speed solar wind during the minimum between cycles 23 and 24. Solar Phys. doi:10.1007/s11207-010-9677-2.

Echer, E., Tsurutani, B.T., Gonzalez, W.D.: 2011. On the use of lowest levels in geomagnetic activity in the space age. Adv. Space Res. submitted.

Echer, E., Tsurutani, B.T., Gonzalez, W.D., Kozyra, J.U.: 2011, High speed stream properties and related geomagnetic activity during the whole heliosphere interval (WHI): 20 March to 16 April 2008. Solar Phys. doi:10.1007/s11207-011-9739-0.

Emery, B.A., Richardson, I.G., Evans, D.S., Rich, F.J., Wilson, G.R.: 2011, Solar rotational periodicities and the semiannual variation in the solar wind, radiation belt, and aurora. Solar Phys. doi:10.1007/s11207-011-9758-x. 
Emmert, J.T., Lean, J.L., Picone, J.M.: 2010, Record-low thermospheric density during the 2008 solar minimum. Geophys. Res. Lett. 37, L12102. doi:10.1029/2010GL043671.

Fisk, L.A., Zhao, L.: 2009, The heliospheric magnetic field and the solar wind during the solar cycle. In: Gopalswamy, N., Webb, D.F. (eds.) Universal Heliophysical Processes, Proc. IAU Symp. 257, Cambridge Univ. Press, Cambridge, 109. doi:10.1017/S1743921309029160.

Galvin, A.B., Kohl, J.L.: 1999, Whole Sun month at solar minimum: An introduction. J. Geophys. Res. 104, 9673. doi:10.1029/1999JA900008.

Gibson, S.E., Biesecker, D.A., Guhathakurta, M., Hoeksema, J.T., Lazarus, A.J., Linker, J., Mikić, Z., Pisanko, Y., Riley, P., Steinberg, J., Strachan, L., Szabo, A., Thompson, B.J., Zhao, X.P.: 1999, The three-dimensional coronal magnetic field during whole Sun month. Astrophys. J. 520, 871. doi:10.1086/307496.

Gibson, S.E., Kozyra, J.U., de Toma, G., Emery, B.A., Onsager, T., Thompson, B.J.: 2009, If the Sun is so quiet, why is the Earth ringing? A comparison of two solar minimum intervals. J. Geophys. Res. 114, 9105. doi:10.1029/2009JA014342.

Gibson, S.E., De Toma, G., Emery, B., Riley, P., Zhao, L., Elsworth, Y., Leamon, R.J., Lei, J., McIntosh, S., Mewaldt, R.A., Thompson, B.J., Webb, D.: 2011, The whole heliosphere interval in the context of a long and structured solar minimum: an overview from Sun to Earth. Solar Phys. doi:10.1007/s11207-011-9921-4

Haber, D.A.: 2008, Subsurface flows near active regions and filaments as determined by local helioseismology. In: Howe, R., Komm, R.W., Balasubramaniam, K.S., Petrie, G.J.D. (eds.) Subsurface and Atmospheric Influences on Solar Activity CS-383, Astron. Soc. Pac., San Francisco, 31.

Haber, D.A., Hindman, B.W., Toomre, J., Bogart, R.S., Larsen, R.M., Hill, F.: 2002, Evolving submerged meridional circulation cells within the upper convection zone revealed by ring-diagram analysis. Astrophys. J. 570, 855. doi:10.1086/339631.

Haberreiter, M.: 2011, Solar EUV spectrum calculated for quiet Sun conditions. Solar Phys. doi:10.1007/ s11207-011-9767-9.

Jackman, C.M., Arridge, C.S.: 2011, Solar cycle effects on the dynamics of Jupiter's and Saturn's magnetospheres. Solar Phys. doi:10.1007/s11207-011-9748-z.

Jackson, B.V., Hick, P.P.: 2005, Three-dimensional tomography of interplanetray disturbances. In: Gary, D., Keller, C. (eds.) Solar and Space Weahther Radiophysics: Current Status and Future Developments, Astrophys. and Space Sci. Lib. 314. Kluwer, Dordrecht, 355. doi:10.1007/1-4020-2814-8_17.

Jian, L.K., Russell, C.T., Luhmann, J.G.: 2011, Comparing solar minimum 23/24 with historical solar wind records at 1 AU. Solar Phys. doi:10.1007/s11207-011-9737-2.

Kosovichev, A.G., Duvall, T.L. Jr.: 2011, Investigation of a sunspot complex by heioseismology. In: Choudhary, D., Strassmeier, K. (eds.) The Physics of Sun and Star Spots, Proc. IAU Symp. 373. Cambridge Univ. Press, Cambridge, 320. doi:10.1017/S1733921311015456.

Leamon, R.J., McIntosh, S.W.: 2009, How the solar wind ties to its photospheric origins. Astrophys. J. Lett. 697, L28. doi:10.1088/0004-637X/697/1/L28.

Lei, J., Thayer, J.P., Wang, W., McPherron, R.L.: 2011, Impact of CIR storms on thermosphere density variability during the solar minimum of 2008. Solar Phys. doi:10.1007/s11207-010-9563-y.

Lepping, R.P., Wu, C.-C., Berdichevsky, D.B., Szabo, A.: 2010, Magnetic clouds at/near the 2007-2009 solar minimum: Frequency of occurrence and some unusual properties. Solar Phys. doi:10.1007/ s11207-010-9646-9.

Linker, J.A., Mikić, Z., Biesecker, D.A., Forsyth, R.J., Gibson, S.E., Lazarus, A.J., Lecinski, A., Riley, P., Szabo, A., Thompson, B.J.: 1999, Magnetohydrodynamic modeling of the solar corona during whole Sun month. J. Geophys. Res. 104, 9809. doi:10.1029/1998JA900159.

Manoharan, P.K.: 2010, Peculiar current solar-minimum structure of the heliosphere. In: Corbett, I.F. (ed.) Whole Heliosphere Interval: Overview of JD16, Highlights of Astronomy 15, Cambridge Univ. Press, Cambridge, 484. doi:10.1017/S1743921310010343.

Maris, G., Maris, O.: 2010, WHI high-speed streams at geospace. In: Corbett, I.F. (ed.) Highlights Astron. 15, Cambridge Univ. Press, Cambridge, 494. doi:10.1017/S147439213100010379.

McIntosh, P.S.: 2003, Patterns and dynamics of solar magnetic fields and He I coronal holes in cycle 23. In: Wilson, A. (ed.) ISCS Symp., Solar Variability as an Input to the Earth's Environment SP-535, ESA, Noordwijk, 807.

McIntosh, S.W., De Pontieu, B.: 2009, What goes up doesn't necessarily come down! Connecting the dynamics of the chromosphere and transition region with TRACE, Hinode and SUMER. In: Lites, B., Cheung, M., Magara, T., Mariska, J., Reeves, K. (eds.) The Second Hinode Science Meeting: Beyond Discovery-Toward Understanding CS-415, Astron. Soc. Pac., San Francisco, 24.

McIntosh, S.W., Davey, A.R., Hassler, D.M.: 2006, Simple magnetic flux balance as an indicator of Ne VIII Doppler velocity partitioning in an equatorial coronal hole. Astrophys. J. Lett. 644, L87. doi:10.1086/505488. 
Mlynczak, M.G., Hunt, L.A., Thomas Marshall, B., Martin-Torres, F.J., Mertens, C.J., Russell, J.M., Remsberg, E.E., López-Puertas, M., Picard, R., Winick, J., Wintersteiner, P., Thompson, R.E., Gordley, L.L.: 2010, Observations of infrared radiative cooling in the thermosphere on daily to multiyear timescales from the TIMED/SABER instrument. J. Geophys. Res. 115, 3309. doi:10.1029/2009JA014713.

Muller, R., Utz, D., Hanslmeier, A.: 2011, Non-varying granulation and photospheric network during the extended 2007 - 2009 solar minimum. Solar Phys. doi:10.1007/s11207-011-9725-6.

Nitta, N.V.: 2011, Observables indicating two major coronal mass ejections during the WHI. Solar Phys. doi:10.1007/s11207-011-9806-6.

Petrie, G.J.D., Canou, A., Amari, T.: 2011, Nonlinear force-free and potential-field models of activeregion and global coronal fields during the whole heliosphere interval. Solar Phys. doi:10.1007/ s11207-010-9687-0.

Picone, J.M., Hedin, A.E., Drob, D.P., Aikin, A.C.: 2002, J. Geophys. Res. 107, 15. doi:10.1029/ 2002JA009430.

Riley, P., Linker, J.A., Mikić, Z.: 2010, Global MHD modeling of the solar corona and inner heliosphere for the whole heliosphere interval. In: Corbett, I.F. (ed.) Whole Heliosphere Interval: Overview of JD16, Highlights of Astronomy 15, Cambridge Univ. Press, Cambridge, 491. doi:10.1017/S1743921310010367.

Riley, P., Lionello, R., Linker, J.A., Mikić, Z., Luhmann, J., Wijaya, J.: 2011, Global MHD modeling of the solar corona and inner heliosphere for the whole heliosphere interval. Solar Phys. doi:10.1007/s11207-010-9698-x.

Russell, C.T., McPherron, R.L.: 1973, Semiannual variation of geomagnetic activity. J. Geophys. Res. 78, 92. doi:10.1029/JA078i001p00092.

Shibasaki, K.: 1998, Radio synoptic maps and polar CAP brightening. In: Balasubramaniam, K.S., Harvey, J., Rabin, D.M. (eds.) Synoptic Solar Physics - 18th NSO/Sacramento Peak Summer Workshop CS-140, Astron. Soc. Pac., San Francisco, 373.

Sojka, J.J., Nicolls, M., van Eyken, A., Heinselman, C., Bilitza, D.: 2011, 24/7 solar minimum polar cap and auroral ion temperature observations. Adv. Space Res. 48, 1. doi:10.1016/j.asr.2011.03.005.

Solomon, S.C., Woods, T.N., Didkovsky, L.V., Emmert, J.T., Qian, L.: 2010, Anomalously low solar extremeultraviolet irradiance and thermospheric density during solar minimum. Geophys. Res. Lett. 37, L16103. doi:10.1029/2010GL044468.

Solomon, S.C., Qian, L., Didkovsky, L.V., Viereck, R.A., Woods, T.N.: 2011, Causes of low thermospheric density during the 2007-2009 solar minimum. J. Geophys. Res. 116, A00H07. doi:10.1029/ 2011JA016508.

Suess, S.T.: 2007, Where is Ulysses today? http://stereo-ssc.nascom.nasa.gov/plans/UlyLocation2006-2014_ v1.pdf, http://ulysses.jpl.nasa.gov/science/UlyLocation2006-2014_v2.pdf.

Thompson, B.J., Gopalswamy, N., Davila, J.M., Haubold, H.J. (eds.): 2009, Putting the "I" in IHY: The United Nations Report for the International Heliophysical Year 2007, Springer, Dordrecht.

Vásquez, A.M., Huang, Z., Manchester, W.B., Frazin, R.A.: 2011, The WHI corona from differential emission measure tomography. Solar Phys. doi:10.1007/s11207-010-9706-1.

Wang, W., Lei, J., Burns, A.G., Qian, L., Solomon, S.C., Wiltberger, M., Xu, J.: 2011, Ionospheric day-to-day variability around the whole heliosphere interval in 2008. Solar Phys. doi:10.1007/s11207-011-9747-0.

Webb, D.F., Cremades, H., Sterling, A.C., Mandrini, C.H., Dasso, S., Gibson, S.E., Haber, D.A., Komm, R.W., Petrie, G.J.D., McIntosh, P.S., Welsch, B.T., Plunkett, S.P.: 2011, The global context of solar activity during the whole heliosphere interval campaign. Solar Phys. doi:10.1007/s11207-011-9787-5.

Welsch, B.T., Christe, S., McTiernan, J.M.: 2011, Photospheric magnetic evolution in the WHI active regions. Solar Phys. doi:10.1007/s11207-011-9759-9.

White, O., Kopp, G., Snow, M., Tapping, K.: 2011, The solar cycle $23-24$ minimum. A benchmark in solar variability and effects in the heliosphere. Solar Phys. doi:10.1007/s11207-010-9680-7.

Woods, T.N., Chamberlin, P.C., Harder, J.W., Hock, R.A., Snow, M., Eparvier, F.G., Fontenla, J., McClintock, W.E., Richard, E.C.: 2009, Solar irradiance reference spectra (SIRS) for the 2008 whole heliosphere interval (WHI). Geophys. Res. Lett. 36, L01101. doi:10.1029/2008GL036373.

Zhao, L., Fisk, L.A.: 2011, Understanding the behavior of the heliospheric magnetic field and the solar wind during the unusual solar minimum between cycles 23 and 24. Solar Phys. doi:10.1007/ s11207-011-9840-4. 